\title{
REMOVING HOLES IN TOPOLOGICAL SHAPE OPTIMIZATION
}

\author{
Philippe Guillaume ${ }^{1}$ and Maatoug Hassine ${ }^{2}$
}

\begin{abstract}
The gradient based topological optimization tools introduced during the last ten years tend naturally to modify the topology of a domain by creating small holes inside the domain. Once these holes have been created, they usually remain unchanged, at least during the topological phase of the optimization algorithm. In this paper, a new asymptotic expansion is introduced which allows to decide whether an existing hole must be removed or not for improving the cost function. Then, two numerical examples are presented: the first one compares topological optimization with standard shape optimization, and the second one, issued from a lake oxygenation problem, illustrates the use of the new asymptotic expansion.
\end{abstract}

Mathematics Subject Classification. 49Q10, 49Q12, 74P05, 74P10, 74P15.

Received December 14, 2005. Revised June 2 and July 19, 2006.

Published online September 21, 2007.

\section{INTRODUCTION}

Topological optimization is concerned with the variation of a cost function with respect to a topology modification of a domain. The most simple way of modifying the topology consists in creating a small hole in the domain. Usually, the cost function involves the solution of a p.d.e. defined on this domain. In the case of structural shape optimization, creating a hole means simply removing some material. In the case of fluid dynamics where the domain represents the fluid, creating a hole means inserting a small obstacle. The situation is similar in electromagnetism. The topological sensitivity tools which have been developed by several authors $[7,8,17,28,30,33,37,38]$ allow to find the place where creating a small hole will bring the best improvement of

\footnotetext{
Keywords and phrases. Topological optimization, topological sensitivity, topological gradient, shape optimization, Stokes equations.

1 MIP, UMR 5640, INSA Département de Mathématiques Complexe Scientifique de Rangueil, 31077 Toulouse Cedex 4, France; philippe.guillaume@insa-toulouse.fr

2 ENIT-LAMSIN et Département de Mathématiques, Faculté des Sciences de Monastir, 5019 Monastir, Tunisia; maatoug.hassine@enit.rnu.tn
}

(c) EDP Sciences, SMAI 2007 
the cost function. These tools are based on a gradient like expression of the form

$$
\begin{gathered}
j\left(\Omega_{\varepsilon}\right)=j(\Omega)+f(\varepsilon) \delta j(x)+o(f(\varepsilon)), \\
f(\varepsilon)>0 \quad \forall \varepsilon>0, \quad \lim _{\varepsilon \rightarrow 0} f(\varepsilon)=0 .
\end{gathered}
$$

Here $\Omega$ is an open and bounded subset of $\mathbb{R}^{d}, d=2,3$, and, for $\varepsilon>0$ and $x \in \Omega, \Omega_{\varepsilon}=\Omega \backslash(\overline{x+\varepsilon \omega})$ is the subset obtained by removing the subset $\overline{x+\varepsilon \omega}$ from $\Omega$, where $\omega \subset \mathbb{R}^{d}$ is a fixed open and bounded subset containing the origin. Obviously, if we want to minimize $j$, the "best" place (in the sense of the steepest descent) where to create an infinitesimal hole is there where $\delta j(x)$ is the most negative. Starting with this observation, topological optimization algorithms can then be constructed [13]. The main problem encountered at this stage is that topological sensitivity only provides information on where to add holes, but not where to remove already existing holes: once a hole has been introduced in the domain, it will remain there during all forthcoming iterations. However, it may happen that after creation of other holes, removing this particular hole would improve the cost function. Hence, there is a need for tools giving an estimate of $j(\Omega)$ when $j\left(\Omega_{\varepsilon}\right)$ is known. An analogy with ordinary differential calculus for a function $u$ is that instead of estimating $u(\varepsilon) \simeq u(0)+\varepsilon u^{\prime}(0)$, we want to estimate $u(0) \simeq u(\varepsilon)-\varepsilon u^{\prime}(\varepsilon)$. Thus, the goal of this paper is to provide and estimate of the form

$$
j(\Omega)=j\left(\Omega_{\varepsilon}\right)-g(x, \varepsilon)+o(f(\varepsilon))
$$

where $g(x, \varepsilon)$ is computed by solving a p.d.e. on the current domain $\Omega_{\varepsilon}$, whereas $\delta j(x)$ in (1) was computed by solving a p.d.e. on the current domain $\Omega$. Formula (1) gives us only the behaviour of $g$ with respect to $\varepsilon$ but cannot be used to compute $g$. During the optimization process, the variations $\delta j$ and $g$ are computed from available data associated to the current domain. A mathematical analysis is given in Section 3.1 for $\delta j$ and in Section 3.2 for $g$. Concerning the hole shape, the theoretical results presented in this paper are valid for any bounded domain $\omega \subset \mathbb{R}^{d}$ containing the origin and having a connected boundary $\partial \omega$ piecewise of class $\mathcal{C}^{1}$. However, in order to get an explicit expression of the boundary integral equation, we will choose a simple geometry: the unit ball.

Other methods like homogenization have been widely used for topological optimization, although they require some penalization procedure in order to retrieve a "classical" domain; see for example $[2,3,14]$ for a recent review on shape optimization. Recently, a new method for modifying the topology has been introduced by Allaire et al. [4], which consists in using a level set method. In contrast to the topological gradient approach which naturally leads to the creation of new holes, the level set approach tends to suppress existing holes. Consequently, the associated optimization algorithm starts with many holes which may gradually disappear (or migrate) during iterations. The new method which is proposed in this paper is a first step towards a method which can both create or suppress holes during the optimization process. The proposed approach is general and can be easily adapted to other partial differential equations like for example elasticity or Helmholtz equations.

The formulation of the problem is presented in Section 2 for the case of Stokes equations with Dirichlet boundary conditions. In Section 3, we first recall the results concerning the creation of a hole and then describe our new result concerning the variation of the cost function when removing a hole. The obtained results are valid for a large class of cost functions and are illustrated in Section 3.4 by two standard examples: the $L^{2}$ and $H^{1}$ distances to a target function. Finally, two numerical examples are presented in Section 4 . The first example is used to compare topological optimization with standard shape optimization. The second example illustrates the use of the new topological asymptotic expansion in a shape optimization problem associated to water eutrophication in a lake. The holes represent air injectors, the position of which must be determined in order to maximize oxygenation of the lake. We will observe that holes are both created and suppressed during the optimization process, and will compare the result with an algorithm where no suppression is allowed. 


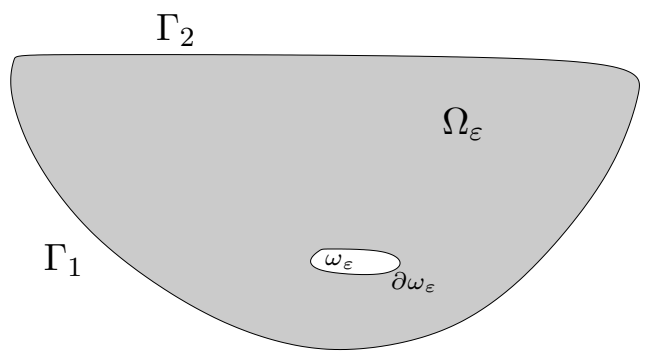

Figure 1. The domain $\Omega_{\varepsilon}=\Omega \backslash \overline{\omega_{\varepsilon}}$.

\section{Formulation of the Problem}

\subsection{Variation of the domain and of the associated p.d.e.}

Let $\Omega_{\varepsilon}$ be a bounded domain of $\mathbb{R}^{d}, d=2,3$, with smooth boundary $\Gamma_{\varepsilon}$, obtained from creating a small hole $\omega_{\varepsilon}$ in a fixed and connected domain $\Omega$. The hole is of the form $\omega_{\varepsilon}=x_{0}+\varepsilon \omega$, where $x_{0} \in \Omega, \varepsilon>0$ and $\omega$ is a given fixed open and bounded domain of $\mathbb{R}^{d}$, containing the origin, whose boundary $\partial \omega$ is connected and piecewise of class $\mathcal{C}^{1}$. It is supposed that $\varepsilon \leq \varepsilon_{0}$ with $\varepsilon_{0}$ sufficiently small so that $\omega_{\varepsilon} \subset \Omega$ for all $\varepsilon \leq \varepsilon_{0}$. The boundary $\Gamma_{\varepsilon}$ satisfies $\Gamma_{\varepsilon}=\Gamma_{1} \cup \Gamma_{2} \cup \partial \omega_{\varepsilon}$ with $\Gamma_{1} \cap \partial \omega_{\varepsilon}=\emptyset$ and $\Gamma_{2} \cap \partial \omega_{\varepsilon}=\emptyset$, where $\Gamma_{1}$ and $\Gamma_{2}$ are portions of $\partial \Omega$ having both a nonnegative Lebesgue measure and satisfy $\Gamma_{1} \cup \Gamma_{2}=\partial \Omega$ and $\Gamma_{1} \cap \Gamma_{2}=\emptyset$ (see Fig. 1).

We consider the Stokes equations describing an incompressible fluid flow in $\Omega_{\varepsilon}$. We denote by $\left(u_{D}^{\varepsilon}, p_{D}^{\varepsilon}\right)$ the solution to the problem with a Dirichlet boundary condition on $\partial \omega_{\varepsilon}$ :

$$
\left\{\begin{array}{rlll}
-\nu \Delta u_{D}^{\varepsilon}+\nabla p_{D}^{\varepsilon} & =0 & & \text { in } \Omega_{\varepsilon} \\
\operatorname{div} u_{D}^{\varepsilon}=0 & & \text { in } \Omega_{\varepsilon} \\
u_{D}^{\varepsilon} & =u_{d} & & \text { on } \Gamma_{1} \\
\nu \partial_{n} u_{D}^{\varepsilon}-p_{D}^{\varepsilon} n & =g & & \text { on } \Gamma_{2} \\
u_{D}^{\varepsilon} & =0 & & \text { on } \partial \omega_{\varepsilon}
\end{array}\right.
$$

where $u_{D}^{\varepsilon}$ is the velocity, $p_{D}^{\varepsilon}$ is the pressure, $\nu$ is the kinematic viscosity of the fluid, $u_{d}$ is a given velocity field on $\Gamma_{1}, I$ is the $d \times d$ identity matrix, $g$ is a given stress vector on $\Gamma_{2}$, and $n$ is the unit outward normal vector along the boundary $\partial \Omega$. For simplicity, no volume forces are considered. Note that for $\varepsilon=0, \Omega_{0}=\Omega$ and $\left(u^{0}, p^{0}\right)$ is solution to

$$
\left\{\begin{aligned}
-\nu \Delta u^{0}+\nabla p^{0} & =0 & & \text { in } \Omega, \\
\operatorname{div} u^{0} & =0 & & \text { in } \Omega, \\
u^{0} & =u_{d} & & \text { on } \Gamma_{1}, \\
\nu \partial_{n} u^{0}-p^{0} n & =g & & \text { on } \Gamma_{2} .
\end{aligned}\right.
$$

For all $\varepsilon \geq 0$, we consider the following Hilbert spaces

$$
\begin{aligned}
& \mathcal{V}_{\varepsilon}=\left\{\theta \in H^{1}\left(\Omega_{\varepsilon}\right)^{d}, \text { div } \theta=0 \text { in } \Omega_{\varepsilon}\right\}, \\
& \mathcal{V}_{\varepsilon}^{0}=\left\{\theta \in \mathcal{V}_{\varepsilon}, \theta=0 \text { on } \partial \omega_{\varepsilon} \text { and } \theta=0 \text { on } \Gamma_{1}\right\},
\end{aligned}
$$


the bilinear form

and the linear form

$$
\begin{aligned}
\mathcal{A}_{\varepsilon}: \mathcal{V}_{\varepsilon} \times \mathcal{V}_{\varepsilon} & \longrightarrow \mathbb{R} \\
(u, w) & \longmapsto \mathcal{A}_{\varepsilon}(u, w)=\nu \int_{\Omega_{\varepsilon}} \nabla u \nabla w \mathrm{~d} x
\end{aligned}
$$

$$
\begin{aligned}
b_{\varepsilon}: \mathcal{V}_{\varepsilon} & \longrightarrow \mathbb{R} \\
w & \longmapsto b_{\varepsilon}(w)=\int_{\Gamma_{2}} g \cdot w \mathrm{~d} s .
\end{aligned}
$$

We have $\mathcal{V}_{\varepsilon}^{0} \subset \mathcal{V}_{\varepsilon} \subset H^{1}\left(\Omega_{\varepsilon}\right)^{d}, \mathcal{A}_{\varepsilon}$ is a bilinear, symmetric, continuous and coercive form on $\mathcal{V}_{\varepsilon}$ and $b_{\varepsilon}$ is a linear and continuous form on $\mathcal{V}_{\varepsilon}$.

\subsubsection{Direct problem}

Let $\Gamma_{1}^{\varepsilon}=\Gamma_{1} \cup \partial \omega_{\varepsilon}$. We denote by $\hat{u}_{d}$ the given boundary data on $\Gamma_{1}^{\varepsilon}$ :

$$
\hat{u}_{d}= \begin{cases}u_{d} & \text { on } \Gamma_{1} \\ 0 & \text { on } \partial \omega_{\varepsilon}\end{cases}
$$

From the weak formulation of the problem (2), we deduce that $u_{D}^{\varepsilon} \in \mathcal{V}_{\varepsilon}$ is solution to

$$
\left\{\begin{array}{l}
\mathcal{A}_{\varepsilon}\left(u_{D}^{\varepsilon}, w\right)=b_{\varepsilon}(w), \quad \forall w \in \mathcal{V}_{\varepsilon}^{0}, \\
u_{D \mid \Gamma_{1}^{\varepsilon}}^{\varepsilon}=\hat{u}_{d}
\end{array}\right.
$$

\subsection{Topological optimization problem}

Consider now a cost function $j(\varepsilon)$ of the form

$$
j(\varepsilon)=J_{\varepsilon}\left(u_{D}^{\varepsilon}\right)
$$

where $J_{\varepsilon}$ is defined on $H^{1}\left(\Omega_{\varepsilon}\right)^{d}$ and satisfies the following hypothesis.

Hypothesis 2.1. There exist a linear and continuous form $L_{\varepsilon}$ defined on $\mathcal{V}_{\varepsilon}$ and a real number $\delta J$ (independent of $\varepsilon$ ) such that

$$
J_{\varepsilon}\left(u_{N}^{\varepsilon}\right)-J_{\varepsilon}\left(u_{D}^{\varepsilon}\right)=L_{\varepsilon}\left(u_{N}^{\varepsilon}-u_{D}^{\varepsilon}\right)+f(\varepsilon) \delta J+o(f(\varepsilon))
$$

where $u_{N}^{\varepsilon} \in \mathcal{V}_{\varepsilon}$ is the solution to the Stokes equations with a Neumann boundary condition on $\partial \omega_{\varepsilon}$ :

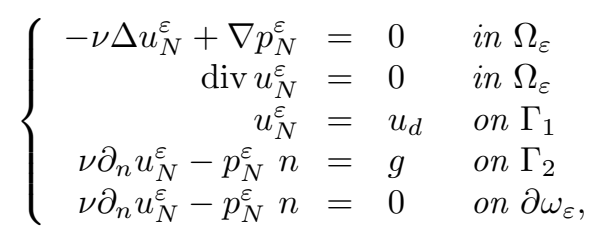

the scalar function $f$ is defined in $\mathbb{R}^{+}$by

$$
f(\varepsilon)= \begin{cases}\varepsilon & \text { if } d=3, \\ -1 / \log (\varepsilon) & \text { if } d=2 .\end{cases}
$$


Remark 2.1. When the function $J_{\varepsilon}(u)$ is differentiable with respect to $u$, the form $L_{\varepsilon}$ coincides with its derivative $D J_{\varepsilon}\left(u_{D}^{\varepsilon}\right)$. The variation $\delta J$ is the leading term of the asymptotic expansion of $J_{\varepsilon}\left(u_{N}^{\varepsilon}\right)-J_{\varepsilon}\left(u_{D}^{\varepsilon}\right)-$ $L_{\varepsilon}\left(u_{N}^{\varepsilon}-u_{D}^{\varepsilon}\right)$ with respect to $\varepsilon$. Some examples are given in Section 3.4 where the form $L_{\varepsilon}$ and the variation $\delta J$ are computed explicitly.

Our aim is to derive an estimate of the cost function $j$ when $\varepsilon$ tends to zero.

\subsubsection{Adjoint problem}

We denote by $v_{D}^{\varepsilon}$ the solution to the adjoint problem associated to (4), that is, $v_{D}^{\varepsilon} \in \mathcal{V}_{\varepsilon}^{0}$ is the solution to

$$
\left\{\begin{array}{l}
v_{D}^{\varepsilon} \in \mathcal{V}_{\varepsilon}^{0}, \\
\mathcal{A}_{\varepsilon}\left(w, v_{D}^{\varepsilon}\right)=-L_{\varepsilon}(w), \quad \forall w \in \mathcal{V}_{\varepsilon}^{0} .
\end{array}\right.
$$

Moreover, due to Rham lemma [18] there exists $q_{D}^{\varepsilon} \in L_{0}^{2}\left(\Omega_{\varepsilon}\right)$ such that

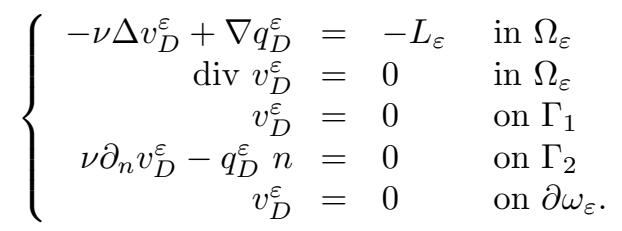

Note that for $\varepsilon=0,\left(v^{0}, q^{0}\right) \in \mathcal{V}_{0}^{0} \times L_{0}^{2}(\Omega)$ is solution to

$$
\left\{\begin{aligned}
-\nu \Delta v^{0}+\nabla q^{0} & =-L_{0} & & \text { in } \Omega \\
\operatorname{div} v^{0} & =0 & & \text { in } \Omega \\
v^{0} & =0 & & \text { on } \Gamma_{1} \\
\nu \partial_{n} v^{0}-q^{0} n & =0 & & \text { on } \Gamma_{2} .
\end{aligned}\right.
$$

\section{VARiation of the COST FUnCtion with RESPECT TO A TOPOLOGICAL PERTURBATION}

As mentioned earlier, the aim of this work is to build a new topological optimization algorithm providing the possibility of creating or suppressing holes during the optimization process. Creation of holes with Dirichlet boundary condition was considered in [22] for the Laplace equation, in [23] for Stokes equations and in [25] for quasi-Stokes equations. For the sake of completeness, we recall in Section 3.1 the results concerning the case where the asymptotic expression of $j(\varepsilon)$ is obtained via computations done on the unperturbed domain $\Omega$.

The main result of this paper is presented in Section 3.3. It concerns the variation of $j(\varepsilon)$ with respect to the suppression of an existing hole. Here, an asymptotic expression for $j(0)$ is obtained via computations done on the perturbed domain $\Omega_{\varepsilon}$. In order to prove this result we give in Section 3.2 a topological sensitivity analysis for the Stokes equations using Neumann boundary condition on the hole, which is also a new result. A topological sensitivity analysis using Neumann boundary condition has already been obtained for the elasticity equations in [17], for Helmholtz equations in [5] and for Maxwell equations in [29]. Here, rather than using a truncation technique, we derive in Section 3.2 a simplified mathematical topological analysis for the Stokes equations.

The results obtained in the two Sections 3.1 and 3.3 will be the basis of a numerical optimization algorithm described in Section 4 and will be used to determine the locations of the holes that will be inserted in or removed from the domain. Finally, we discuss Hypothesis 2.1 in Section 3.4, and we compute the variation of the cost function for two standards examples: the $L^{2}$ and $H^{1}$ distances to a given target function. 


\subsection{Creating a small hole using Dirichlet condition}

The topological sensitivity analysis for the Stokes equations when creating a small hole inside the domain with a Dirichlet boundary condition is considred in [23]. We recall here the main results of this case. We distinguish the cases $d=2$ and $d=3$, this is due to the fact that the fundamental solutions to the Stokes equations in $\mathbb{R}^{2}$ and $\mathbb{R}^{3}$ have an essentially different asymptotic behavior at infinity. It is proved in [23] that $f(\varepsilon)=\varepsilon$ if $d=3$ and $f(\varepsilon)=-1 / \log (\varepsilon)$ if $d=2$.

3.1.1. The three dimensional case

Theorem 3.1. If Hypothesis 2.1 holds, then the function $j$ given in (5) has the following asymptotic expansion

$$
j(\varepsilon)=j(0)+\varepsilon\left[\left(-\int_{\partial \omega} T(y) \mathrm{d} s(y)\right) \cdot v^{0}\left(x_{0}\right)+\delta J\right]+o(\varepsilon) .
$$

The function $T$ is a density associated to a single layer potential representation (see e.g. [16]), of an exterior Stokes problem solution defined in $\mathbb{R}^{3} \backslash \bar{\omega}$. Then, $T \in H^{-1 / 2}(\partial \omega)^{d}$ is solution to the following boundary integral equation, for more detail one can see [23] or [25]

$$
\int_{\partial \omega} E(x-y) T(y) \mathrm{d} s(y)=-u^{0}\left(x_{0}\right), \quad \forall x \in \partial \omega
$$

The function $v^{0}$ is the solution to the adjoint problem $(10)$, and the function $(E, P)$ is the fundamental solution to the Stokes equations in $3 D$

$$
E(y)=\frac{1}{8 \pi \nu r}\left(I+e_{r} e_{r}^{T}\right), \quad P(y)=\frac{y}{4 \pi r^{3}},
$$

with $r=\|y\|, e_{r}=y / r$ and $e_{r}^{T}$ is the transposed vector of $e_{r}$.

In the particular case where $\omega$ is the unit ball $B(0,1)$, we have

$$
\int_{\partial \omega} E(x-y) \mathrm{d} s(y)=\frac{2}{3 \nu} I, \quad \forall x \in \partial \omega
$$

Hence, the density $T$ is given explicitly

$$
T(y)=\frac{3 \nu}{2} u^{0}\left(x_{0}\right), \quad \forall y \in \partial \omega .
$$

Corollary 3.1. Let $x_{0} \in \Omega$ and $\omega=B(0,1)$. Under the hypotheses of Theorem 3.1 , we have

$$
j(\varepsilon)=j(0)+\varepsilon\left[6 \pi \nu u^{0}\left(x_{0}\right) \cdot v^{0}\left(x_{0}\right)+\delta J\right]+o(\varepsilon) .
$$

3.1.2. The two dimensional case

In this case the fundamental solution $(E, P)$ of the Stokes equations is given by

$$
E(y)=\frac{1}{4 \pi \nu}\left(-\log (r) I+e_{r} e_{r}^{T}\right), \quad P(y)=\frac{y}{2 \pi r^{2}}
$$


Theorem 3.2. Under the same hypotheses of Theorem 3.1, the function $j$ has the following asymptotic expansion

$$
j(\varepsilon)=j(0)-1 / \log (\varepsilon)\left[4 \pi \nu u^{0}\left(x_{0}\right) \cdot v^{0}\left(x_{0}\right)+\delta J\right]+o(-1 / \log (\varepsilon)) .
$$

\subsection{Creating a small hole using Neumann condition}

We establish in this section an asymptotic expansion of the cost function $\tilde{j}$ associated to the Stokes equations with a Neumann condition on the boundary of the hole. Here $\tilde{j}$ is defined by

$$
\tilde{j}(\varepsilon)=J_{\varepsilon}\left(u_{N}^{\varepsilon}\right)
$$

where $u_{N}^{\varepsilon}$ is solution to (7).

From the weak formulation of (7) one can show that $u_{N}^{\varepsilon} \in \mathcal{V}_{\varepsilon}$ is solution to

$$
\left\{\begin{array}{l}
\mathcal{A}_{\varepsilon}\left(u_{N}^{\varepsilon}, w\right)=b_{\varepsilon}(w), \quad \forall w \in \mathcal{V}_{N}^{0, \varepsilon} \\
u_{N \mid \Gamma_{1}}^{\varepsilon}=u_{d}
\end{array}\right.
$$

where $\mathcal{V}_{N}^{0, \varepsilon}=\left\{\theta \in \mathcal{V}_{\varepsilon}, \quad \theta_{\mid \Gamma_{1}}=0\right\}$

Next, we suppose that $J_{\varepsilon}$ satisfies the following hypothesis.

Hypothesis 3.1. For all $\varepsilon \geq 0$, there exist a linear form $L_{N}^{\varepsilon}$ on $\mathcal{V}_{\varepsilon}$ and a real number $\delta J_{N}$ such that

$$
J_{\varepsilon}\left(u_{N}^{\varepsilon}\right)-J_{0}\left(u^{0}\right)=L_{N}^{\varepsilon}\left(u_{N}^{\varepsilon}-u^{0}\right)+\varepsilon^{d} \delta J_{N}+o\left(\varepsilon^{d}\right)
$$

For more detail concerning this hypothesis one can see [5]. We denote by $v_{N}^{\varepsilon}$ the solution to the associated adjoint problem

$$
\left\{\begin{array}{l}
v_{N}^{\varepsilon} \in \mathcal{V}_{N}^{0, \varepsilon} \\
\mathcal{A}_{\varepsilon}\left(w, v_{N}^{\varepsilon}\right)=-L_{N}^{\varepsilon}(w), \quad \forall w \in \mathcal{V}_{N}^{0, \varepsilon}
\end{array}\right.
$$

Moreover, using Rham lemma [18] one can prove that there exists $q_{N}^{\varepsilon} \in L_{0}^{2}\left(\Omega_{\varepsilon}\right)$ such that

$$
\left\{\begin{aligned}
-\nu \Delta v_{N}^{\varepsilon}+\nabla q_{N}^{\varepsilon} & =-L_{N}^{\varepsilon} & & \text { in } \Omega_{\varepsilon} \\
\operatorname{div} v_{N}^{\varepsilon} & =0 & & \text { in } \Omega_{\varepsilon} \\
v_{N}^{\varepsilon} & =0 & & \text { on } \Gamma_{1} \\
\nu \partial_{n} v_{N}^{\varepsilon}-q_{N}^{\varepsilon} n & =0 & & \text { on } \Gamma_{2} \\
\nu \partial_{n} v_{N}^{\varepsilon}-q_{N}^{\varepsilon} n & =0 & & \text { on } \partial \omega_{\varepsilon}
\end{aligned}\right.
$$

Our aim is to derive the behavior of $\tilde{j}$ with respect to $\varepsilon$.

From (17), we have

Using hypothesis 3.1 , we obtain

$$
\tilde{j}(\varepsilon)-\tilde{j}(0)=J_{\varepsilon}\left(u_{N}^{\varepsilon}\right)-J_{0}\left(u^{0}\right)
$$

$$
\tilde{j}(\varepsilon)-\tilde{j}(0)=L_{N}^{\varepsilon}\left(u_{N}^{\varepsilon}-u^{0}\right)+\varepsilon^{d} \delta J_{N}+o\left(\varepsilon^{d}\right) .
$$

Using (20), we derive

$$
\tilde{j}(\varepsilon)-\tilde{j}(0)=\mathcal{A}_{\varepsilon}\left(u^{0}-u_{N}^{\varepsilon}, v_{N}^{\varepsilon}\right)+\varepsilon^{d} \delta J_{N}+o\left(\varepsilon^{d}\right) .
$$

In the following section, we give an estimate of the term $\mathcal{A}_{\varepsilon}\left(u^{0}-u_{N}^{\varepsilon}, v_{N}^{\varepsilon}\right)$. To this end, we need to complete the hypothesis 3.1 by the following assumption. 
Hypothesis 3.2. There exist a function $L_{N}$ of regularity $\mathcal{C}^{0} \cap H^{1}$ in a neighborhood of $x_{0}$ such that for all $u \in H^{1}(\Omega)$ and all $\varepsilon \geq 0$,

$$
L_{0}(u)=L_{N}^{\varepsilon}\left(u_{\mid \Omega_{\varepsilon}}\right)+\int_{\omega_{\varepsilon}} L_{N} u \mathrm{~d} x
$$

Note that using this hypothesis and equation (10) one can prove that $\left(v^{0}, q^{0}\right)$ (weakly) satisfies the following system:

$$
\left\{\begin{aligned}
-\nu \Delta v^{0}+\nabla q^{0} & =-L_{N} & & \text { in } \omega_{\varepsilon} \\
\operatorname{div} v^{0} & =0 & & \text { in } \omega_{\varepsilon}
\end{aligned}\right.
$$

\subsubsection{Preliminary estimates}

From (3) and (7), we have

$$
\begin{aligned}
\mathcal{A}_{\varepsilon}\left(u^{0}-u_{N}^{\varepsilon}, v_{N}^{\varepsilon}\right) & =\nu \int_{\Omega_{\varepsilon}} \nabla\left(u^{0}-u_{N}^{\varepsilon}\right) \nabla v_{N}^{\varepsilon} \mathrm{d} x=\int_{\partial \omega_{\varepsilon}}\left(\nu \partial_{n} u^{0}-p^{0} n\right) \cdot v_{N}^{\varepsilon} \mathrm{d} s \\
& =\int_{\partial \omega_{\varepsilon}}\left(\nu \partial_{n} u^{0}-p^{0} n\right) \cdot v^{0} \mathrm{~d} s+\int_{\partial \omega_{\varepsilon}}\left(\nu \partial_{n} u^{0}-p^{0} n\right) \cdot\left(v_{N}^{\varepsilon}-v^{0}\right) \mathrm{d} s .
\end{aligned}
$$

In the following, we give an estimate of each term. The following lemma gives an estimate for the first one.

Lemma 3.1. We have

$$
\begin{aligned}
\int_{\partial \omega_{\varepsilon}}\left(\nu \partial_{n} u^{0}-p^{0} n\right) \cdot v^{0} \mathrm{~d} s=\int_{\partial \omega_{\varepsilon}}\left(\nu \partial_{n} v^{0}-q^{0} n\right)\left(x_{0}\right) \cdot\left(u^{0}-u^{0}\left(x_{0}\right)\right) \mathrm{d} s+\rho_{1}(\varepsilon)+\rho_{2}(\varepsilon), \\
\text { with } \rho_{1}(\varepsilon)=\int_{\partial \omega_{\varepsilon}}\left(\left(\nu \partial_{n} v^{0}-q^{0} n\right)-\left(\nu \partial_{n} v^{0}-q^{0} n\right)\left(x_{0}\right)\right) \cdot\left(u^{0}-u^{0}\left(x_{0}\right)\right) \mathrm{d} s, \\
\rho_{2}(\varepsilon)=-\int_{\omega_{\varepsilon}} L_{N}\left(u^{0}-u^{0}\left(x_{0}\right)\right) \mathrm{d} x .
\end{aligned}
$$

Proof. Using Green formula, from (3) we have

$$
\int_{\partial \omega_{\varepsilon}}\left(\nu \partial_{n} u^{0}-p^{0} n\right) \cdot v^{0} \mathrm{~d} s=\nu \int_{\omega_{\varepsilon}} \nabla u^{0} \nabla v^{0} \mathrm{~d} x=\nu \int_{\omega_{\varepsilon}} \nabla\left(u^{0}-u^{0}\left(x_{0}\right)\right) \nabla v^{0} \mathrm{~d} x .
$$

Using equation (22) we deduce

$$
\nu \int_{\omega_{\varepsilon}} \nabla\left(u^{0}-u^{0}\left(x_{0}\right)\right) \nabla v^{0} \mathrm{~d} x=\int_{\partial \omega_{\varepsilon}}\left(\nu \partial_{n} v^{0}-q^{0} n\right) \cdot\left(u^{0}-u^{0}\left(x_{0}\right)\right) \mathrm{d} s-\int_{\omega_{\varepsilon}} L_{N}\left(u^{0}-u^{0}\left(x_{0}\right)\right) \mathrm{d} x
$$

We now examine the second term of (23). Let $w_{\varepsilon}=v_{N}^{\varepsilon}-v^{0}$ and $\xi_{\varepsilon}=q_{N}^{\varepsilon}-q^{0}$. Using Hypothesis 3.2, from (21) we deduce that $\left(w_{\varepsilon}, \xi_{\varepsilon}\right)$ is solution to

$$
\left\{\begin{aligned}
-\nu \Delta w_{\varepsilon}+\nabla \xi_{\varepsilon} & =0 & & \text { in } \Omega_{\varepsilon} \\
\operatorname{div} w_{\varepsilon} & =0 & & \text { in } \Omega_{\varepsilon} \\
w_{\varepsilon} & =0 & & \text { on } \Gamma_{1} \\
\nu \partial_{n} w_{\varepsilon}-\xi_{\varepsilon} n & =0 & & \text { on } \Gamma_{2} \\
\nu \partial_{n} w_{\varepsilon}-\xi_{\varepsilon} n & =-\left(\nu \partial_{n} v^{0}-q^{0} n\right) & & \text { on } \partial \omega_{\varepsilon}
\end{aligned}\right.
$$


Due to the regularity of $v^{0}$ and $q^{0}$ one can approximate $\left(w_{\varepsilon}, \xi_{\varepsilon}\right)$ by $\left(\widetilde{w}_{\varepsilon}, \widetilde{\xi}_{\varepsilon}\right)$ solution to the same system with a constant right hand side

$$
\left\{\begin{aligned}
-\nu \Delta \widetilde{w}_{\varepsilon}+\nabla \widetilde{\xi}_{\varepsilon} & =0 & & \text { in } \Omega_{\varepsilon} \\
\operatorname{div} \widetilde{w}_{\varepsilon} & =0 & & \text { in } \Omega_{\varepsilon} \\
\widetilde{w}_{\varepsilon} & =0 & & \text { on } \Gamma_{1} \\
\nu \partial_{n} \widetilde{w}_{\varepsilon}-\widetilde{\xi}_{\varepsilon} n & =0 & & \text { on } \Gamma_{2} \\
\nu \partial_{n} \widetilde{w}_{\varepsilon}-\widetilde{\xi}_{\varepsilon} n & =-\left(\nu \partial_{n} v^{0}-q^{0} n\right)\left(x_{0}\right) & & \text { on } \partial \omega_{\varepsilon} .
\end{aligned}\right.
$$

Let the change of variable

$$
\widetilde{w}(x)=1 / \varepsilon \widetilde{w}_{\varepsilon}(\varepsilon x), \quad \widetilde{\xi}(x)=\widetilde{\xi}_{\varepsilon}(\varepsilon x), \quad \forall x \in \mathbb{R}^{d} \backslash \bar{\omega} .
$$

It is easy to see that $(\widetilde{w}, \widetilde{\xi})$ is a solution to

$$
\left\{\begin{aligned}
-\nu \Delta \widetilde{w}+\nabla \widetilde{\xi} & =0 & & \text { in } \mathbb{R}^{d} \backslash \bar{\omega} \\
\operatorname{div} \widetilde{w} & =0 & & \text { in } \mathbb{R}^{d} \backslash \bar{\omega} \\
\nu \partial_{n} \widetilde{w}-\widetilde{\xi} n & =-\left(\nu \partial_{n} v^{0}-q^{0} n\right)\left(x_{0}\right) & & \text { on } \partial \omega .
\end{aligned}\right.
$$

The function $\widetilde{w}$ is the leading term with respect to $\varepsilon$ of the variation $v_{N}^{\varepsilon}-v^{0}$. Using the regularity of $v^{0}$ and $q^{0}$ one can prove that $v_{N}^{\varepsilon}(x)-v^{0}(x)=\varepsilon \widetilde{w}(x / \varepsilon)+o(\varepsilon)$.

Using the simple layer potential $[16],(\widetilde{w}, \widetilde{\xi})$ can be written as

$$
\widetilde{w}(y)=\int_{\partial \omega} E(y-x) \eta(x) \mathrm{d} s(x), \quad \widetilde{\xi}(y)=\int_{\partial \omega} P(y-x) \cdot \eta(x) \mathrm{d} s(x), \quad y \in \mathbb{R}^{d} \backslash \bar{\omega}
$$

where $E, P$ is the fundamental solution of the Stokes equations (see (13) and (15)).

The function $\eta \in H^{-1 / 2}(\partial \omega)^{d}$ is the solution to the boundary integral equation (see e.g. [16])

$$
-\frac{\eta(y)}{2}+\int_{\partial \omega}\left(\nabla_{y}(E(x-y) \eta(x))-P(x-y) \cdot \eta(x)\right) \cdot n(y) \mathrm{d} s(x)=-\left(\nu \partial_{n(y)} v^{0}-q^{0} n(y)\right)\left(x_{0}\right), \quad \forall y \in \partial \omega,
$$

subscript $y$ in $\nabla_{y}$ denoting a differentiation with respect to $y$.

Next, for all $\varphi \in H^{1 / 2}\left(\partial \omega_{\varepsilon}\right)$, such that $\int_{\partial \omega_{\varepsilon}} \varphi \cdot n \mathrm{~d} s=0$, we denote by $\left(h_{\varepsilon}^{\varphi}, \xi_{\varepsilon}^{\varphi}\right) \in H^{1}\left(\omega_{\varepsilon}\right) \times L_{0}^{2}\left(\omega_{\varepsilon}\right)$ the solution to

$$
\left\{\begin{array}{rll}
-\nu \Delta h_{\varepsilon}^{\varphi}+\nabla \xi_{\varepsilon}^{\varphi}= & 0 & \text { in } \omega_{\varepsilon} \\
\operatorname{div} h_{\varepsilon}^{\varphi}= & 0 & \text { in } \omega_{\varepsilon} \\
h_{\varepsilon}^{\varphi}= & \varphi & \text { on } \partial \omega_{\varepsilon}
\end{array}\right.
$$

Denoting

$$
\begin{aligned}
& \rho_{3}(\varepsilon)=\int_{\partial \omega_{\varepsilon}}\left(\nu \partial_{n}\left(h_{\varepsilon}^{w_{\varepsilon}}-h_{\varepsilon}^{\tilde{w}_{\varepsilon}}\right)-\left(\xi_{\varepsilon}^{w_{\varepsilon}}-\xi_{\varepsilon}^{\tilde{w}_{\varepsilon}}\right) n\right) \cdot\left(u^{0}(x)-u^{0}\left(x_{0}\right)\right) \mathrm{d} s, \\
& \rho_{4}(\varepsilon)=-\varepsilon^{d-1} \int_{\partial \omega} \eta(y) \cdot\left(u^{0}\left(x_{0}+\varepsilon y\right)-u^{0}\left(x_{0}\right)-\nabla u^{0}\left(x_{0}\right)(\varepsilon y)\right) \mathrm{d} s(y) .
\end{aligned}
$$

We have the following result.

\section{Lemma 3.2.}

$\int_{\partial \omega_{\varepsilon}}\left(\nu \partial_{n} u^{0}-p^{0} n\right) \cdot w_{\varepsilon} \mathrm{d} s=-\varepsilon^{d} \nabla u^{0}\left(x_{0}\right) \int_{\partial \omega} \eta(y) \cdot y \mathrm{~d} s(y)-\int_{\partial \omega_{\varepsilon}}\left(\nu \partial_{n} v^{0}-q^{0} n\right)\left(x_{0}\right) \cdot\left(u^{0}-u^{0}\left(x_{0}\right)\right) \mathrm{d} s+\rho_{3}(\varepsilon)+\rho_{4}(\varepsilon)$. 
Proof. From (3), we have

$$
\int_{\partial \omega_{\varepsilon}}\left(\nu \partial_{n} u^{0}-p^{0} n\right) \cdot w_{\varepsilon} \mathrm{d} s=\nu \int_{\omega_{\varepsilon}} \nabla\left(u^{0}(x)-u^{0}\left(x_{0}\right)\right) \nabla h_{\varepsilon}^{w_{\varepsilon}} \mathrm{d} x .
$$

Using the fact that $\left(h_{\varepsilon}^{w_{\varepsilon}}, \xi_{\varepsilon}^{w_{\varepsilon}}\right)$ is solution to

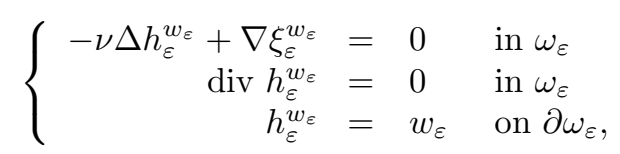

we obtain

$$
\begin{aligned}
\int_{\partial \omega_{\varepsilon}}\left(\nu \partial_{n} u^{0}-p^{0} n\right) \cdot w_{\varepsilon} \mathrm{d} s= & \int_{\partial \omega_{\varepsilon}}\left(\nu \partial_{n}\left(h_{\varepsilon}^{w_{\varepsilon}}-h_{\varepsilon}^{\widetilde{w}_{\varepsilon}}\right)-\left(\xi_{\varepsilon}^{w_{\varepsilon}}-\xi_{\varepsilon}^{\widetilde{w}_{\varepsilon}}\right) n\right) \cdot\left(u^{0}(x)-u^{0}\left(x_{0}\right)\right) \mathrm{d} s \\
& +\int_{\partial \omega_{\varepsilon}}\left(\nu \partial_{n} h_{\varepsilon}^{\widetilde{w}_{\varepsilon}}-\xi_{\varepsilon}^{\widetilde{w}_{\varepsilon}} n\right) \cdot\left(u^{0}(x)-u^{0}\left(x_{0}\right)\right) \mathrm{d} s .
\end{aligned}
$$

Observe that $\left(h^{\widetilde{w}}, \xi^{\widetilde{w}}\right)$ is solution to

$$
\left\{\begin{aligned}
-\nu \Delta h^{\widetilde{w}}+\nabla \xi^{\widetilde{w}} & =0 & & \text { in } \omega \\
\operatorname{div} h^{\widetilde{w}} & =0 & & \text { in } \omega \\
\nu \partial_{n} h^{\widetilde{w}}-\xi^{\widetilde{w}} n & =-\left(\nu \partial_{n} v^{0}-q^{0} n\right)\left(x_{0}\right) & & \text { on } \partial \omega
\end{aligned}\right.
$$

and satisfies the relations

$$
\forall x \in \omega, \quad h^{\widetilde{w}}(x)=(1 / \varepsilon) h_{\varepsilon}^{\widetilde{w}_{\varepsilon}}(\varepsilon x), \text { and }\left(\nu \nabla h^{\widetilde{w}} \cdot n(x)-\xi^{\widetilde{w}} n(x)\right)(x)=\left(\nu \nabla h_{\varepsilon}^{\widetilde{w}_{\varepsilon}} \cdot n(\varepsilon x)-\xi_{\varepsilon}^{\widetilde{w}_{\varepsilon}} n(\varepsilon x)\right)(\varepsilon x) .
$$

Taking into account that the density $\eta$ is the stress tensor jump on $\partial \omega$, we have

$$
\eta(x)=-\left(\nu \partial_{n} v^{0}-q^{0} n\right)\left(x_{0}\right)-\left(\nu \partial_{n} h^{\widetilde{w}}-\xi^{\widetilde{w}} n\right), \forall x \in \omega .
$$

Then, the second term of (29) can be expressed as

$$
\begin{aligned}
\int_{\partial \omega_{\varepsilon}}\left(\nu \partial_{n} h_{\varepsilon}^{\widetilde{w}_{\varepsilon}}-\xi_{\varepsilon}^{\widetilde{w}_{\varepsilon}} n\right) \cdot\left(u^{0}(x)-u^{0}\left(x_{0}\right)\right) \mathrm{d} s= & -\int_{\partial \omega_{\varepsilon}} \eta(x / \varepsilon) \cdot\left(u^{0}(x)-u^{0}\left(x_{0}\right)\right) \mathrm{d} s(x) \\
& -\int_{\partial \omega_{\varepsilon}}\left(\nu \partial_{n} v^{0}-q^{0} n\right)\left(x_{0}\right) \cdot\left(u^{0}(x)-u^{0}\left(x_{0}\right)\right) \mathrm{d} s(x) \\
= & -\varepsilon^{d} \int_{\partial \omega} \eta(y) \cdot \nabla u^{0}\left(x_{0}\right) y \mathrm{~d} s(y)+\rho_{4}(\varepsilon) \\
& -\int_{\partial \omega_{\varepsilon}}\left(\nu \partial_{n} v^{0}-q^{0} n\right)\left(x_{0}\right) \cdot\left(u^{0}(x)-u^{0}\left(x_{0}\right)\right) \mathrm{d} s(x)
\end{aligned}
$$

Finally, substituting the last equation in (29) and using Lemma 3.1, we obtain the wanted result.

Proposition 3.1. The bilinear form $\mathcal{A}_{\varepsilon}$ has the following expansion

$$
\mathcal{A}_{\varepsilon}\left(u^{0}-u_{N}^{\varepsilon}, v_{N}^{\varepsilon}\right)=-\varepsilon^{d} \int_{\partial \omega} \eta(y) \cdot \nabla u^{0}\left(x_{0}\right) y \mathrm{~d} s(y)+\sum_{j=1,4} \rho_{j}(\varepsilon) .
$$




\subsubsection{Asymptotic expansion}

The asymptotic expansion of $\tilde{j}$ for Stokes equations with Neumann condition on the boundary of the hole is given for a large class of cost functions by the following theorem.

Theorem 3.3. Let $\tilde{j}(\varepsilon)=J_{\varepsilon}\left(u_{N}^{\varepsilon}\right)$ a cost function satisfying the Hypothesis 3.1. Then, under the assumptions 3.2 , the function $\tilde{j}$ has the following asymptotic expansion:

$$
\tilde{j}(\varepsilon)=\tilde{j}(0)+\varepsilon^{d}\left(-\int_{\partial \omega} \eta(y) \cdot \nabla u^{0}\left(x_{0}\right) y \mathrm{~d} s(y)+\delta J_{N}\right)+o\left(\varepsilon^{d}\right) .
$$

Proof. From Lemma 3.1, Lemma 3.2 and Proposition 3.1, it is sufficient to show that

$$
\rho_{j}(\varepsilon)=o\left(\varepsilon^{d}\right), \quad \text { for all } j=1, \ldots, 4 \text {. }
$$

Such estimates can be proved using a change of variable, a Taylor expansion around $x_{0}$ and the regularity of $u^{0}$, $p^{0}, v^{0}$ and $q^{0}$ near $x_{0}$. For a similar technique, we refer to [5].

\subsection{Variation of the cost function when removing a small hole}

We are now in position to compute the variation of the cost function when removing a small hole $\omega_{\varepsilon}$. The main result is presented in Theorem 3.4. The principal term of the variation is described by an integral on the boundary of the hole $\omega_{\varepsilon}$. It has naturally the same behavior with respect to $\varepsilon$ than the variation issued from the creation of a hole. Using the same technique as in $[22,23,25]$, one can prove that this term is of order $\varepsilon$ in the three dimensional case and of order $-1 / \log (\varepsilon)$ in the two dimensional case.

The expression in Theorem 3.4 involves the normal derivative of the Dirichlet adjoint solution $v_{D}^{\varepsilon}$ (see Eq. (8)) and the solution $u_{N}^{\varepsilon}$ to the Stokes problem with a Neumann boundary condition on $\partial \omega_{\varepsilon}$ (see Eq. (7)). The latter was introduced as an auxiliary problem. Using Green's formula, one can show that it is also possible to write the same term by using the normal derivative of $u_{D}^{\varepsilon}$ and the adjoint solution $v_{N}^{\varepsilon}$ associated to the Neumann Stokes problem.

Theorem 3.4. If Hypothesis 2.1 holds, then we have the following estimate:

$$
j(0)=j(\varepsilon)+\int_{\partial \omega_{\varepsilon}}\left(\nu \partial_{n} v_{D}^{\varepsilon}-q_{D}^{\varepsilon} n\right) \cdot u_{N}^{\varepsilon} \mathrm{d} s+f(\varepsilon) \delta J+o(f(\varepsilon)) .
$$

Proof. Using the asymptotic expansion of $\tilde{j}$ established in the previous section and the fact that $\varepsilon^{d}=o(f(\varepsilon))$ and $\tilde{j}(0)=j(0)$ we derive a first estimate of the variation of $j$ :

$$
\begin{aligned}
j(0)-j(\varepsilon) & =\tilde{j}(\varepsilon)-j(\varepsilon)+o(f(\varepsilon)), \\
& =J_{\varepsilon}\left(u_{N}^{\varepsilon}\right)-J_{\varepsilon}\left(u_{D}^{\varepsilon}\right)+o(f(\varepsilon)) .
\end{aligned}
$$

Due to Hypothesis 2.1, we have

$$
j(0)-j(\varepsilon)=L_{\varepsilon}\left(u_{N}^{\varepsilon}-u_{D}^{\varepsilon}\right)+f(\varepsilon) \delta J+o(f(\varepsilon)) .
$$

Using (9) and the fact that $u_{D \mid \partial \omega_{\varepsilon}}^{\varepsilon}=0$, we deduce

$$
L_{\varepsilon}\left(u_{N}^{\varepsilon}-u_{D}^{\varepsilon}\right)=\nu \int_{\Omega_{\varepsilon}} \nabla v_{D}^{\varepsilon} \nabla\left(u_{D}^{\varepsilon}-u_{N}^{\varepsilon}\right) \mathrm{d} x+\int_{\partial \omega_{\varepsilon}}\left(\nu \partial_{n} v_{D}^{\varepsilon}-q_{D}^{\varepsilon} n\right) \cdot u_{N}^{\varepsilon} \mathrm{d} s
$$


Taking into account $v_{D \mid \Gamma_{1}}^{\varepsilon}=0$ and $v_{D \mid \partial \omega_{\varepsilon}}^{\varepsilon}=0$, from (2), we have

$$
\nu \int_{\Omega_{\varepsilon}} \nabla v_{D}^{\varepsilon} \nabla u_{D}^{\varepsilon} \mathrm{d} x=\int_{\Gamma_{2}} g \cdot v_{D}^{\varepsilon} \mathrm{d} s
$$

From (7), we obtain

$$
\nu \int_{\Omega_{\varepsilon}} \nabla v_{D}^{\varepsilon} \nabla u_{N}^{\varepsilon} \mathrm{d} x=\int_{\Gamma_{2}} g \cdot v_{D}^{\varepsilon} \mathrm{d} s
$$

Then,

Hence, we derive

$$
L\left(u_{D}^{\varepsilon}\right)\left(u_{N}^{\varepsilon}-u_{D}^{\varepsilon}\right)=\int_{\partial \omega_{\varepsilon}}\left(\nu \partial_{n} v_{D}^{\varepsilon}-q_{D}^{\varepsilon} n\right) \cdot u_{N}^{\varepsilon} \mathrm{d} s
$$

$$
j(0)-j(\varepsilon)=\int_{\partial \omega_{\varepsilon}}\left(\nu \partial_{n} v_{D}^{\varepsilon}-q_{D}^{\varepsilon} n\right) \cdot u_{N}^{\varepsilon} \mathrm{d} s+f(\varepsilon) \delta J+o(f(\varepsilon)) .
$$

\subsection{Cost function examples}

We now discuss Hypothesis 2.1, we present two standards examples of cost function satisfying this hypothesis and we compute their variations $\delta J$.

\subsubsection{First example}

Proposition 3.2. Consider the cost function

$$
J_{\varepsilon}(u)=\int_{\Omega_{\varepsilon}}|u-\mathcal{U}|^{2} \mathrm{~d} x,
$$

where $\mathcal{U} \in H^{1}(\Omega)$. Then, Hypothesis 2.1 holds with

$$
L_{\varepsilon}(w)=2 \int_{\Omega_{\varepsilon}}\left(u_{D}^{\varepsilon}-\mathcal{U}\right) \cdot w \mathrm{~d} x, \quad \forall w \in \mathcal{V}_{\varepsilon}, \text { and } \delta J=0 .
$$

Proof. We have

$$
\begin{aligned}
J_{\varepsilon}\left(u_{N}^{\varepsilon}\right)-J_{\varepsilon}\left(u_{D}^{\varepsilon}\right) & =\int_{\Omega_{\varepsilon}}\left|u_{N}^{\varepsilon}-\mathcal{U}\right|^{2} \mathrm{~d} x-\int_{\Omega_{\varepsilon}}\left|u_{D}^{\varepsilon}-\mathcal{U}\right|^{2} \mathrm{~d} x \\
& =2 \int_{\Omega_{\varepsilon}}\left(u_{D}^{\varepsilon}-\mathcal{U}\right) \cdot\left(u_{N}^{\varepsilon}-u_{D}^{\varepsilon}\right) \mathrm{d} x+\left\|u_{D}^{\varepsilon}-u_{N}^{\varepsilon}\right\|_{0, \Omega_{\varepsilon}}^{2}
\end{aligned}
$$

The triangular inequality yields

$$
\left\|u_{D}^{\varepsilon}-u_{N}^{\varepsilon}\right\|_{0, \Omega_{\varepsilon}} \leq\left\|u_{D}^{\varepsilon}-u^{0}\right\|_{0, \Omega_{\varepsilon}}+\left\|u_{N}^{\varepsilon}-u^{0}\right\|_{0, \Omega_{\varepsilon}} .
$$

It is proved in [23] that

$$
\left\|u_{D}^{\varepsilon}-u^{0}\right\|_{0, \Omega_{\varepsilon}}=O(f(\varepsilon))
$$


By an adaptation of the technique described in [17] one can prove that

$$
\left\|u_{N}^{\varepsilon}-u^{0}\right\|_{0, \Omega_{\varepsilon}}=O\left(\varepsilon^{d}\right)=o(f(\varepsilon)) .
$$

Thus, we have

$$
\left\|u_{D}^{\varepsilon}-u_{N}^{\varepsilon}\right\|_{0, \Omega_{\varepsilon}}^{2}=o(f(\varepsilon))
$$

and it follows from (38) and (39) that

$$
J_{\varepsilon}\left(u_{D}^{\varepsilon}\right)-J_{\varepsilon}\left(u_{N}^{\varepsilon}\right)=2 \int_{\Omega_{\varepsilon}}\left(u_{D}^{\varepsilon}-\mathcal{U}\right) \cdot\left(u_{N}^{\varepsilon}-u_{D}^{\varepsilon}\right) \mathrm{d} x+o(f(\varepsilon)) .
$$

Consequently, we have $\delta J=0$ and $L_{\varepsilon}(w)=2 \int_{\omega}\left(u_{D}^{\varepsilon}-\mathcal{U}\right) \cdot w \mathrm{~d} x, \quad \forall w \in \mathcal{V}_{\varepsilon}$.

\subsubsection{Second example}

Proposition 3.3. Consider the cost function

$$
J_{\varepsilon}(u)=\nu \int_{\Omega_{\varepsilon}}|\nabla u-\nabla \mathcal{U}|^{2} \mathrm{~d} x,
$$

where $\mathcal{U} \in H^{1}(\Omega)$. Then, Hypothesis 2.1 holds with

$$
\begin{aligned}
& L_{\varepsilon}(w)=2 \int_{\Omega_{\varepsilon}} \nabla\left(u_{D}^{\varepsilon}-\mathcal{U}\right) \nabla w \mathrm{~d} x \quad \forall w \in \mathcal{V}_{\varepsilon}, \\
& \delta J= \begin{cases}\left(-\int_{\partial \omega} T(y) \mathrm{d} s(y)\right) \cdot u^{0}\left(x_{0}\right) & \text { if } d=3, \\
4 \pi \nu\left|u^{0}\left(x_{0}\right)\right|^{2} & \text { if } d=2 .\end{cases}
\end{aligned}
$$

For $d=3$, if $\omega$ is the unit ball $B(0,1)$, we have

$$
\delta J=6 \pi \nu\left|u^{0}\left(x_{0}\right)\right|^{2} .
$$

Proof. From the definition (40) we derive

$$
\begin{aligned}
J_{\varepsilon}\left(u_{N}^{\varepsilon}\right)-J_{\varepsilon}\left(u_{D}^{\varepsilon}\right) & =\nu \int_{\Omega_{\varepsilon}}\left|\nabla u_{N}^{\varepsilon}-\nabla \mathcal{U}\right|^{2} \mathrm{~d} x-\nu \int_{\Omega_{\varepsilon}}\left|\nabla u_{D}^{\varepsilon}-\nabla \mathcal{U}\right|^{2} \mathrm{~d} x \\
& =2 \nu \int_{\Omega_{\varepsilon}}\left(\nabla u_{D}^{\varepsilon}-\nabla \mathcal{U}\right)\left(\nabla u_{N}^{\varepsilon}-\nabla u_{D}^{\varepsilon}\right) \mathrm{d} x+\nu \int_{\Omega_{\varepsilon}}\left|\nabla u_{D}^{\varepsilon}-\nabla u_{N}^{\varepsilon}\right|^{2} \mathrm{~d} x .
\end{aligned}
$$

To estimate the term $\int_{\Omega_{\varepsilon}}\left|\nabla u_{D}^{\varepsilon}-\nabla u_{N}^{\varepsilon}\right|^{2} \mathrm{~d} x$, we need to distinguish the 2D case from the 3D case. We only discuss the $3 \mathrm{D}$ case, the approach for the $2 \mathrm{D}$ case being similar.

First, we consider the following decomposition:

$$
\nu \int_{\Omega_{\varepsilon}}\left|\nabla u_{D}^{\varepsilon}-\nabla u_{N}^{\varepsilon}\right|^{2} \mathrm{~d} x=\nu\left|u_{D}^{\varepsilon}-u^{0}\right|_{1, \Omega_{\varepsilon}}^{2}+\nu\left|u_{N}^{\varepsilon}-u^{0}\right|_{1, \Omega_{\varepsilon}}^{2}-2 \nu \int_{\Omega_{\varepsilon}}\left(\nabla u_{D}^{\varepsilon}-\nabla u^{0}\right)\left(\nabla u_{N}^{\varepsilon}-\nabla u^{0}\right) \mathrm{d} x
$$


Next, we derive an asymptotic expansion with respect to $\varepsilon$ for each term of the last equality.

- Estimate of the first term $\nu\left|u_{D}^{\varepsilon}-u^{0}\right|_{1, \Omega_{\varepsilon}}^{2}$ :

We split $u_{D}^{\varepsilon}-u^{0}$ into $u_{\varepsilon}^{1}+u_{\varepsilon}^{2}$ and their associated pressure $p_{D}^{\varepsilon}-p^{0}$ into $p_{\varepsilon}^{1}+p_{\varepsilon}^{2}$ such that

$$
\left\{\begin{array} { r l r l } 
{ - \nu \Delta u _ { \varepsilon } ^ { 1 } + \nabla p _ { \varepsilon } ^ { 1 } } & { = 0 } & { } & { \text { in } \mathbb { R } ^ { 3 } \backslash \overline { \omega _ { \varepsilon } } } \\
{ \operatorname { d i v } u _ { \varepsilon } ^ { 1 } = 0 } & { } & { \text { in } \mathbb { R } ^ { 3 } \backslash \overline { \omega _ { \varepsilon } } } \\
{ u _ { \varepsilon } ^ { 1 } = 0 } & { \text { at } \infty } \\
{ u _ { \varepsilon } ^ { 1 } = - u ^ { 0 } ( x _ { 0 } ) } & { \text { on } \partial \omega _ { \varepsilon } , }
\end{array} \quad \left\{\begin{array}{rlrl}
-\nu \Delta u_{\varepsilon}^{2}+\nabla p_{\varepsilon}^{2} & =0 & \text { in } \Omega_{\varepsilon} \\
\operatorname{div} u_{\varepsilon}^{2} & =0 & \text { in } \Omega_{\varepsilon} \\
u_{\varepsilon}^{2} & =-u_{\varepsilon}^{1} & \text { on } \Gamma_{1} \\
\nu \partial_{n} u_{\varepsilon}^{2}-p_{\varepsilon}^{2} n & =-\left(\nu \partial_{n} u_{\varepsilon}^{1}-p_{\varepsilon}^{1} n\right) & \text { on } \Gamma_{2} \\
u_{\varepsilon}^{2} & =-u^{0}+u^{0}\left(x_{0}\right) & \text { on } \partial \omega_{\varepsilon}
\end{array}\right.\right.
$$

We consider the change of variable,

$$
U(x)=u_{\varepsilon}^{1}(\varepsilon x), \quad P(x)=\varepsilon p_{\varepsilon}^{1}(\varepsilon x)
$$

Then $(U, P)$ is the solution to the Stokes exterior problem

$$
\left\{\begin{aligned}
-\nu \Delta U+\nabla P & =0 & & \text { in } \mathbb{R}^{3} \backslash \bar{\omega} \\
\operatorname{div} U & =0 & & \text { in } \mathbb{R}^{3} \backslash \bar{\omega} \\
U & =0 & & \text { at } \infty \\
U & =-u^{0}\left(x_{0}\right) & & \text { on } \partial \omega .
\end{aligned}\right.
$$

Using the potential simple layer $[16],(U, P)$ can be written as

$$
U(y)=\int_{\partial \omega} E(y-x) T(x) \mathrm{d} s(x), P(y)=\int_{\partial \omega} P(y-x) \cdot T(x) \mathrm{d} s(x), \quad \forall y \in \mathbb{R}^{3} \backslash \bar{\omega} .
$$

From the previous decomposition, we have

$$
\nu\left|u_{D}^{\varepsilon}-u^{0}\right|_{1, \Omega_{\varepsilon}}^{2}=\nu \int_{\Omega_{\varepsilon}} \nabla u_{\varepsilon}^{1} \nabla u_{\varepsilon}^{1} \mathrm{~d} x+2 \nu \int_{\Omega_{\varepsilon}} \nabla u_{\varepsilon}^{1} \nabla u_{\varepsilon}^{2} \mathrm{~d} x+\nu \int_{\Omega_{\varepsilon}} \nabla u_{\varepsilon}^{2} \nabla u_{\varepsilon}^{2} \mathrm{~d} x .
$$

Due to Green Formula and the change of variable, from (43) we derive

$$
\nu \int_{\Omega_{\varepsilon}} \nabla u_{\varepsilon}^{1} \nabla u_{\varepsilon}^{1} \mathrm{~d} x=\int_{\partial \omega_{\varepsilon}}\left(\nu \partial_{n} u_{\varepsilon}^{1}-p_{\varepsilon}^{1} n\right) \cdot u_{\varepsilon}^{1} \mathrm{~d} s=-\varepsilon \int_{\partial \omega}\left(\nu \partial_{n} U-P n\right) \cdot u^{0}\left(x_{0}\right) \mathrm{d} s .
$$

By jump relation (see [16]), we have $\nu \partial_{n} U-P n(x)=T(x), \quad \forall x \in \partial \omega$.

Then,

$$
\nu \int_{\Omega_{\varepsilon}} \nabla u_{\varepsilon}^{1} \nabla u_{\varepsilon}^{1} \mathrm{~d} x=-\varepsilon\left(\int_{\partial \omega} T(x) \mathrm{d} s\right) \cdot u^{0}\left(x_{0}\right) .
$$

To estimate the second and the third term of (44), we need the following lemma.

Lemma $3.3([17])$. For $\phi \in H_{V}^{1 / 2}(\partial \omega)^{3}$; let $z, s$ be the solution to the Stokes exterior problem

$$
\left\{\begin{array}{rlll}
-\nu \Delta z+\nabla s & =0 & & \text { in } \mathbb{R}^{3} \backslash \bar{\omega} \\
\operatorname{div} z & =0 & & \text { in } \mathbb{R}^{3} \backslash \bar{\omega} \\
z & =0 & & \text { at infinity } \\
z & =\phi & & \text { on } \partial \omega .
\end{array}\right.
$$


There exists a constant $c>0$, independent of $\phi$ and $\varepsilon$, such that

$$
\begin{aligned}
\|z\|_{0, C(R /(2 \varepsilon), R / \varepsilon)} & \leq c \varepsilon^{-1 / 2}\|\phi\|_{1 / 2, \partial \omega} \\
|z|_{1, C(R /(2 \varepsilon), R / \varepsilon)} & \leq c \varepsilon^{1 / 2}\|\phi\|_{1 / 2, \partial \omega} .
\end{aligned}
$$

with $R>0$, such that $\omega_{\varepsilon} \subset B(0, R)$ and $B(0, R) \subset \Omega$.

Then due to this Lemma and a change of variable, we deduce that

$$
\left\|u_{\varepsilon}^{1}\right\|_{1, \Omega_{R}} \leq c \varepsilon
$$

By elliptic regularity and trace theorem, we have

$$
\left\|u_{\varepsilon}^{2}\right\|_{1, \Omega_{\varepsilon}} \leq c\left\{\left\|u_{\varepsilon}^{1}\right\|_{1 / 2, \Gamma_{1}}+\left\|\nu \partial_{n} u_{\varepsilon}^{1}-p_{\varepsilon}^{1} n\right\|_{-1 / 2, \Gamma_{2}}\right\} \leq c\left\|u_{\varepsilon}^{1}\right\|_{1, \Omega_{R}} .
$$

Finally, using Hölder's inequality, from (45), (46) and (47) we obtain

$$
\nu\left|u_{D}^{\varepsilon}-u^{0}\right|_{1, \Omega_{\varepsilon}}^{2}=-\varepsilon\left(\int_{\partial \omega} T(x) \mathrm{d} s\right) \cdot u^{0}\left(x_{0}\right)+o(\varepsilon)
$$

- Estimate of the second term $\nu\left|u_{N}^{\varepsilon}-u^{0}\right|_{1, \Omega_{\varepsilon}}^{2}$ :

We split $u_{N}^{\varepsilon}-u^{0}$ into $w_{\varepsilon}^{1}+w_{\varepsilon}^{2}$ and their associated pressure $p_{N}^{\varepsilon}-p^{0}$ into $s_{\varepsilon}^{1}+s_{\varepsilon}^{2}$ such that

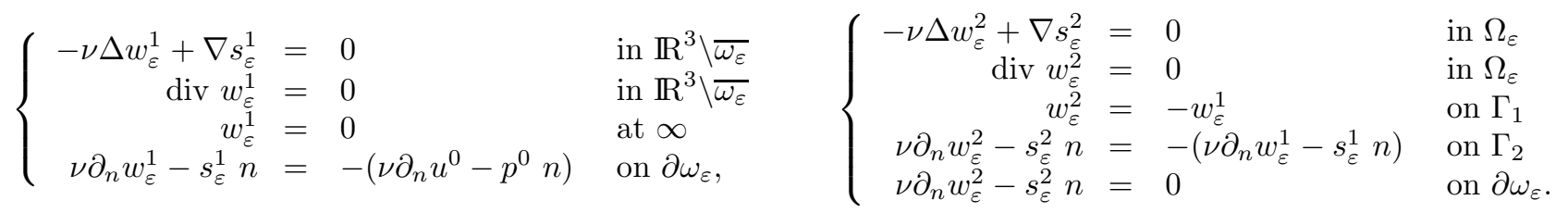

We consider the change of variable,

$$
W_{\varepsilon}(x)=w_{\varepsilon}^{1}(\varepsilon x), \quad S_{\varepsilon}(x)=\varepsilon s_{\varepsilon}^{1}(\varepsilon x)
$$

Then $\left(W_{\varepsilon}, S_{\varepsilon}\right)$ is the solution to the Stokes exterior problem

$$
\left\{\begin{aligned}
-\nu \Delta W_{\varepsilon}+\nabla S_{\varepsilon} & =0 & & \text { in } \mathbb{R}^{3} \backslash \bar{\omega} \\
\operatorname{div} W_{\varepsilon} & =0 & & \text { in } \mathbb{R}^{3} \backslash \bar{\omega} \\
W_{\varepsilon} & =0 & & \text { at } \infty \\
\nu \partial_{n} W_{\varepsilon}-S_{\varepsilon} n & =-\varepsilon\left(\nu \partial_{n} u^{0}-p^{0} n\right) & & \text { on } \partial \omega .
\end{aligned}\right.
$$

The same technique described in previous section can be adapted for the Neumann case. One can prove here that

$$
\nu\left|u_{N}^{\varepsilon}-u^{0}\right|_{1, \Omega_{\varepsilon}}^{2}=o(\varepsilon)
$$

Fore a similar work, one can see [17] where some estimates are derived in the elasticity equations case. 
- Estimate of the third term $\nu \int_{\Omega_{\varepsilon}}\left(\nabla u_{D}^{\varepsilon}-\nabla u^{0}\right)\left(\nabla u_{N}^{\varepsilon}-\nabla u^{0}\right) \mathrm{d} x$ :

Using Hölder's inequality, it follow from (48) and (49) that

$$
\nu \int_{\Omega_{\varepsilon}}\left(\nabla u_{D}^{\varepsilon}-\nabla u^{0}\right)\left(\nabla u_{N}^{\varepsilon}-\nabla u^{0}\right) \mathrm{d} x=o(\varepsilon) .
$$

Finally from (41), (48), (49) and (50), we deduce the required result.

In the particular case where $\omega$ is the unit ball $B(0,1)$, we have $T(y)=\frac{3 \nu}{2} u^{0}\left(x_{0}\right), \forall y \in \partial \omega$.

Hence, $\delta J=6 \pi \nu\left|u^{0}\left(x_{0}\right)\right|^{2}$.

\section{Numerical RESUlts}

This section presents two numerical examples. The first one compares topological optimization with standard shape optimization, whereas the second one illustrates the new topological asymptotic expansion.

\subsection{Example 1: comparison between classical and topological gradient methods}

The problem which is here considered consists in finding in the domain $\Omega$ the optimal location of a small hole $\omega_{\varepsilon}$ in order to reach a given target flow $\mathcal{U}_{g}$. The optimization problem that we consider can be formulated as follows.

\section{Optimization problem $(\mathcal{P})$ :}

For a given $\varepsilon>0$, find the position of $\omega_{\varepsilon}$ in $\Omega$ minimizing the cost function

$$
J\left(u_{\varepsilon}, \Omega \backslash \bar{\omega}_{\varepsilon}\right)=\int_{\Omega \backslash \bar{\omega}_{\varepsilon}}\left|u_{\varepsilon}-\mathcal{U}_{g}\right|^{2} \mathrm{~d} x
$$

where $\left(u_{\varepsilon}, p_{\varepsilon}\right)$ is solution to the Stokes equations in $\Omega_{\varepsilon}=\Omega \backslash \bar{\omega}_{\varepsilon}$

$$
\left\{\begin{aligned}
-\nu \Delta u_{\varepsilon}+\nabla p_{\varepsilon} & =0 & & \text { in } \Omega_{\varepsilon}, \\
\operatorname{div} u_{\varepsilon} & =0 & & \text { in } \Omega_{\varepsilon}, \\
u_{\varepsilon} & =u_{d} & & \text { on } \partial \Omega, \\
u_{\varepsilon} & =u_{i n j} & & \text { on } \partial \omega_{\varepsilon},
\end{aligned}\right.
$$

and $u_{d}$ and $u_{i n j}$ are given boundary data.

We will treat this problem using two different numerical approaches. The first approach is presented in Section 4.1.1. It considers $(\mathcal{P})$ as a classical shape optimization problem. The second approach is the topological gradient method. It is presented in Section 4.1.2. For the numerical computation, we will use the following data.

The given data: We consider a small circular hole $\omega_{\varepsilon}=X_{0}+\varepsilon B(0,1)$ with $B(0,1)$ is the unit ball and $\varepsilon=0.02$. The computational domain $\Omega$ is a square with edge length equal to one. On the top edge a constant positive horizontal velocity is prescribed $\left(u_{d}=(0.1,0)\right)$. The other edges are regarded as walls with homogeneous Dirichlet boundary conditions. On the variable boundary $\partial \omega_{\varepsilon}$ a constant vertical velocity is imposed $\left(u_{i n j}=(0,0.5)\right)$. The wanted flow $\mathcal{U}_{g}$ is chosen as the solution to system (52) with $\omega_{\varepsilon}$ centered at the point $X_{0}=(0.3,0.4)$ (see Fig. 2$)$. 


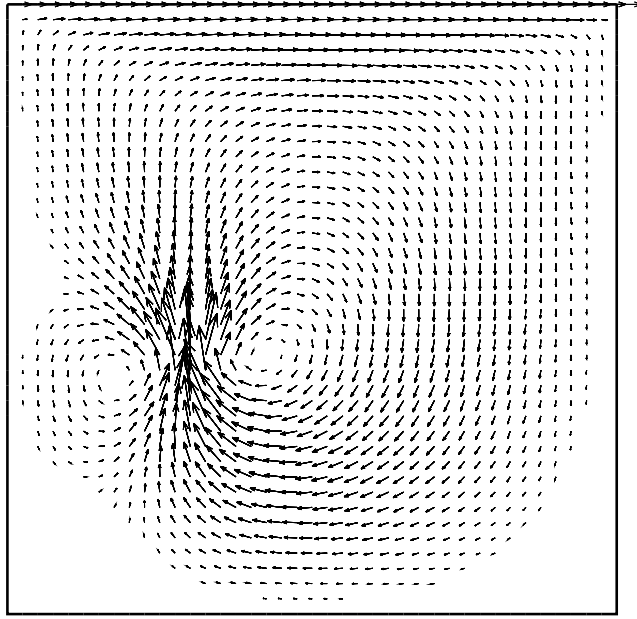

Figure 2. The wanted flow $\mathcal{U}_{g}$.

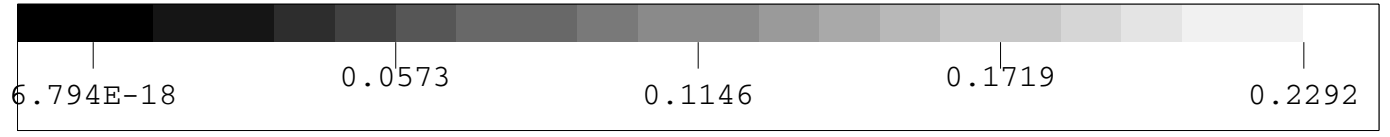
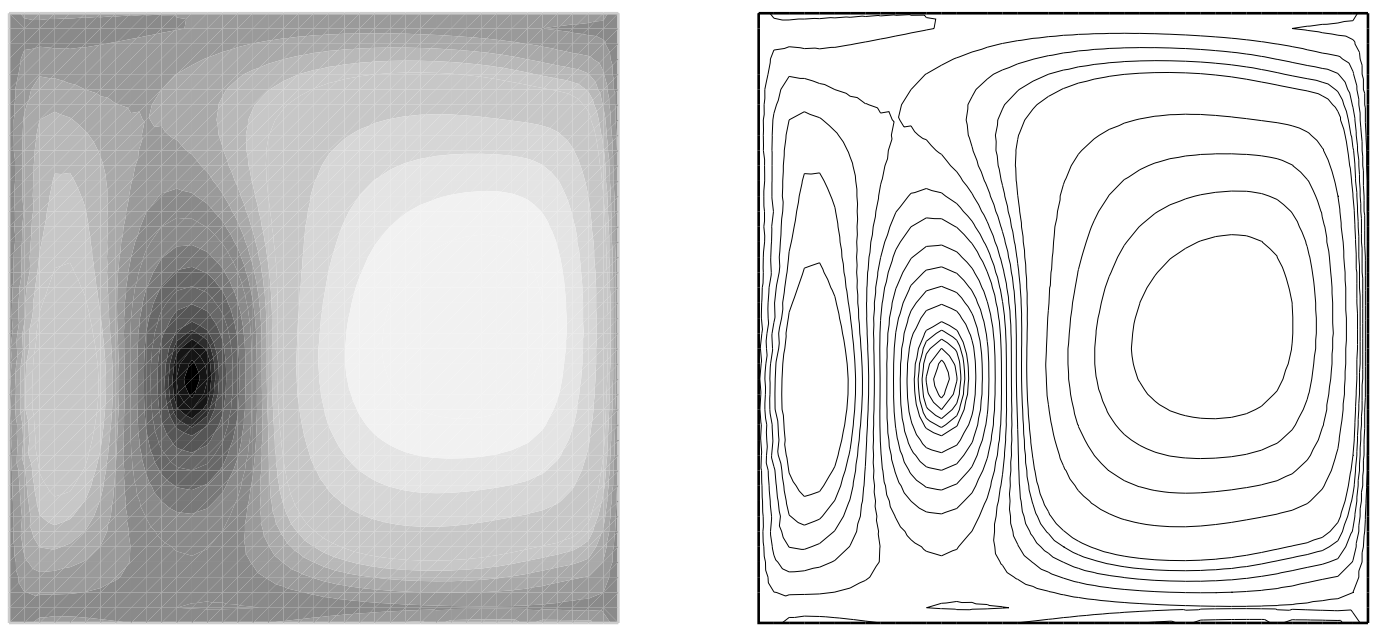

Figure 3 . The cost function $J$ to be minimized.

Using the previous data, we have computed the cost function $J$ at each mesh node $X_{i}$ :

$$
J\left(X_{i}\right)=\int_{\Omega \backslash \bar{\omega}_{\varepsilon}^{i}}\left|u_{\varepsilon}^{i}-\mathcal{U}_{g}\right|^{2} \mathrm{~d} x
$$

where $\omega_{\varepsilon}^{i}=X_{i}+\varepsilon B(0,1)$ and $u_{\varepsilon}^{i}$ is the solution to (52) with $\omega_{\varepsilon}=\omega_{\varepsilon}^{i}$. The obtained result is plotted in Figure 3 . One can see that $J$ has several local minima (near the top and bottom edges) and of course a global minimum at the point $X=(0.3,0.4)$. 


\subsubsection{Shape optimization gradient method}

The problem $(\mathcal{P})$ can be viewed as a classical domain optimization problem where the unknown is the position of $\partial \omega_{\varepsilon}$, a part of the boundary of the computational domain $\Omega_{\varepsilon}$. Domain optimization for the Stokes equations were studied, for example, by Pironneau [31] who computed the shape of body with minimum drag. Gunzburger and Kim [24] showed existence of an optimal shape for a minimum drag problem in a channel flow. Simon et al. [6] proved differentiability of the drag with respect to domain variations in Navier-Stokes flow. We also refer to $[20,21,32]$ where derivatives (first, second and higher order) of a function with respect to the variation of the domain are analyzed for different operators.

The modified flow domain $\Omega_{\varepsilon}^{t}$ is of the form

$$
\Omega_{\varepsilon}^{t}=\Omega \backslash \overline{\omega_{\varepsilon}^{t}}, \omega_{\varepsilon}^{t}=(I+t V)\left(\omega_{\varepsilon}\right)
$$

with $V \in W^{1, \infty}\left(\Omega, \mathbb{R}^{2}\right)$ and $t \in \mathbb{R}$. It is supposed that $V(x)=0$ for $x \in \partial \Omega$. As we here consider only translations of $\omega_{\varepsilon}, V(x)$ is constant on $\partial \omega_{\varepsilon}$. Then, using the Lagrangian method, the first order derivative of $J$ with respect to the domain variation $V$ is given by

$$
D J\left(u_{\varepsilon}, \Omega_{\varepsilon}^{0}\right) V=-\int_{\partial \omega_{\varepsilon}} \frac{\partial u_{\varepsilon}}{\partial n} \cdot \frac{\partial v_{\varepsilon}}{\partial n} V \cdot n \mathrm{~d} s,
$$

where $u_{\varepsilon}$ is solution to $(52)$ and $v_{\varepsilon}$ is solution to the associated adjoint problem (for more details we refer to $[20,35,36])$.

Obtained results: We have used a minimization algorithm based on the quasi-Newton method. Table 1 summarizes the results obtained by using six different initial positions $X_{0}^{k}, 1 \leq k \leq 6$. We describe in this table, for each case, the obtained position, the iterations and simulations number and the cost function values. One can observe that the algorithm converges to the exact solution when the initial position $X_{0} \in\left\{X_{0}^{2}, X_{0}^{3}, X_{0}^{4}\right\}$. When $X_{0} \in\left\{X_{0}^{1}, X_{0}^{5}, X_{0}^{6}\right\}$, it converges to a local minimum.

We present in Figure 4, for each initial position choice, the position obtained during the optimization process. Figure 5 gives the values of the cost function in the three cases $X_{0} \in\left\{X_{0}^{2}, X_{0}^{3}, X_{0}^{4}\right\}$ where we have convergence to the exact solution.

\subsubsection{Topological optimization gradient method}

The topological gradient method provides an asymptotic expansion of the cost function with respect to a small topological perturbation of the domain. Taking into account that we consider here the Stokes equations with non homogeneous boundary condition on $\partial \omega_{\varepsilon}\left(u_{\varepsilon}=u_{i n j}\right)$, we deduce from Theorem 3.2 and Proposition 3.2 the following expansion of the function $j(\varepsilon)=J\left(u_{\varepsilon}, \Omega \backslash \bar{\omega}_{\varepsilon}\right)$ :

$$
j(\varepsilon)=j(0)-\frac{1}{\log (\varepsilon)}\left[4 \pi \nu\left(u^{0}(x)-u_{i n j}\right) \cdot v^{0}(x)\right]+o\left(\frac{-1}{\log (\varepsilon)}\right),
$$

where $u^{0}$ and $v^{0}$ are respectively solution to

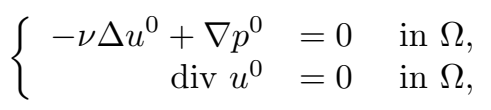

and

$$
\left\{\begin{aligned}
-\nu \Delta v^{0}+\nabla q^{0} & =-2\left(u^{0}-\mathcal{U}_{g}\right) & & \text { in } \Omega \\
\operatorname{div} v^{0} & =0 & & \text { in } \Omega .
\end{aligned}\right.
$$


TABLE 1. Obtained results for each initial position $X_{0}^{k}, k=1, . ., 6$.

\begin{tabular}{|c|c|c|c|c|}
\hline Initial position & Obtained position & $\begin{array}{c}\text { Iterations and } \\
\text { simulations number }\end{array}$ & $\begin{array}{c}\text { Initial value } \\
\text { of } J\end{array}$ & $\begin{array}{c}\text { Final value } \\
\text { of } J\end{array}$ \\
\hline \hline$X_{0}^{1}=(0.1,0.8)$ & $\begin{array}{c}\text { converge to local minimum } \\
Y^{1}=(0.02999,0.97000)\end{array}$ & $\begin{array}{c}3 \text { iterations, } \\
14 \text { simulations }\end{array}$ & $0.92968 \mathrm{E}-01$ & $0.73337 \mathrm{E}-01$ \\
\hline$X_{0}^{2}=(0.2,0.7)$ & $\begin{array}{c}\text { converge to exact solution } \\
Y^{2}=(0.29999,0.40007)\end{array}$ & $\begin{array}{c}10 \text { iterations, } \\
19 \text { simulations }\end{array}$ & $0.82249 \mathrm{E}-01$ & $0.95734 \mathrm{E}-05$ \\
\hline$X_{0}^{3}=(0.3,0.1)$ & $\begin{array}{c}\text { converge to exact solution } \\
Y^{3}=(0.29999,0.40006)\end{array}$ & $\begin{array}{c}5 \text { iterations, } \\
14 \text { simulations }\end{array}$ & $0.69252 \mathrm{E}-01$ & $0.86717 \mathrm{E}-05$ \\
\hline$X_{0}^{4}=(0.5,0.6)$ & $\begin{array}{c}\text { converge to exact solution } \\
Y^{4}=(0.30000,0.40007)\end{array}$ & $\begin{array}{c}9 \text { iterations, } \\
15 \text { simulations }\end{array}$ & $0.13931 \mathrm{E}+00$ & $0.89545 \mathrm{E}-05$ \\
\hline$X_{0}^{5}=(0.75,0.4)$ & $\begin{array}{c}\text { converge to local minimum } \\
Y^{5}=(0.32816,0.02999)\end{array}$ & $\begin{array}{c}4 \text { iterations, } \\
46 \text { simulations }\end{array}$ & $0.22156 \mathrm{E}+00$ & $0.68282 \mathrm{E}-01$ \\
\hline$X_{0}^{6}=(0.8,0.6)$ & $\begin{array}{c}3 \text { iterations, } \\
\text { converge to local minimum } \\
Y^{6}=(0.97000,0.97010)\end{array}$ & $\begin{array}{c}43 \text { simulations } \\
\text { nat }\end{array}$ & $0.21347 \mathrm{E}+00$ & $0.74261 \mathrm{E}-01$ \\
\hline
\end{tabular}

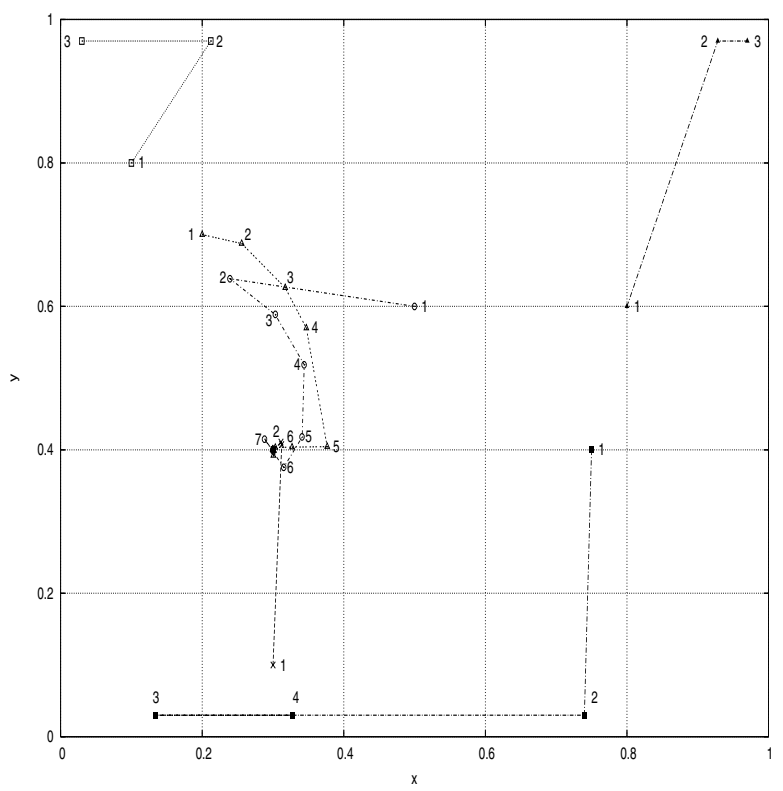

Figure 4. Positions obtained during optimization process for each initial position $X_{0}^{k}, k=1, \ldots, 6$.

We recall here the topological optimization algorithm introduced by Céa, Gioan and Michel [12] and presented in the topological asymptotic context in $[5,13,17,22,23,25]$. 


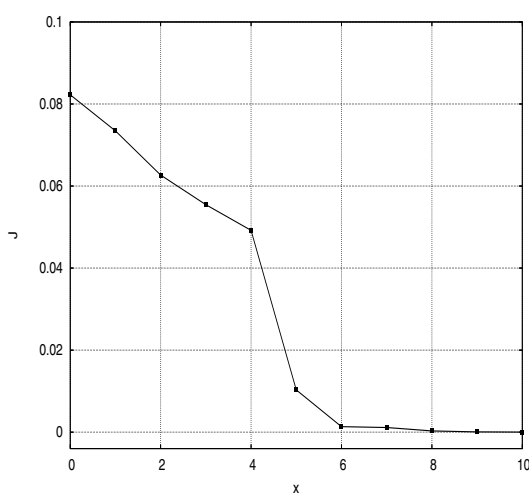

(a) case $X_{0}=(0.2,0.7)$.

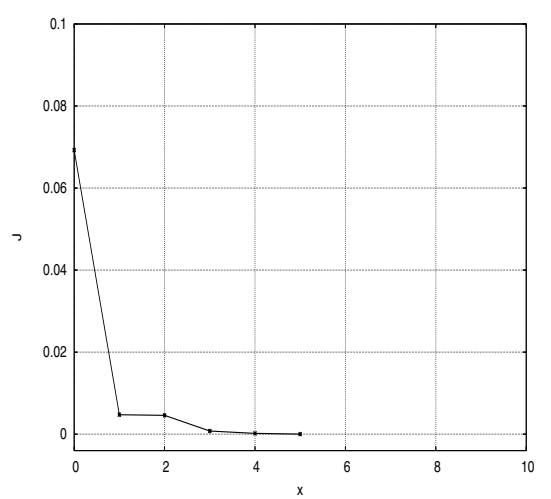

(b) case $X_{0}=(0.3,0.1)$.

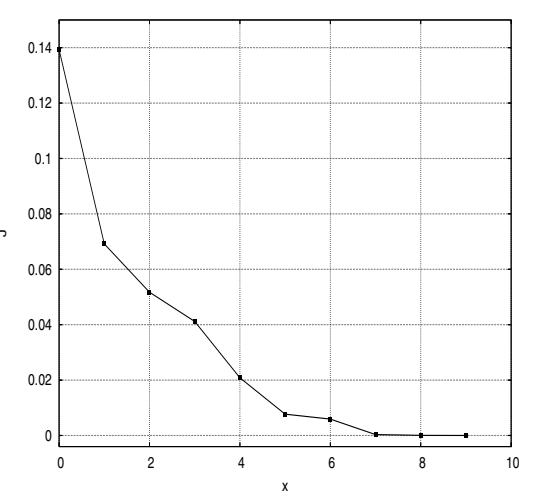

(c) case $X_{0}=(0.5,0.6)$.

Figure 5. Values of the cost function $J$.

\section{Algorithm 1.}

- Initialization: choose $\Omega_{0}$ and set $k=0$.

- Repeat until target is reached:

- solve (53) and (54) in $\Omega_{k}$;

- compute the topological sensitivity $\delta j_{k}$;

- set $\Omega_{k+1}=\left\{x \in \Omega_{k}, \quad \delta j_{k}(x) \geq c_{k}\right\}$ where $c_{k}$ is chosen in such a way that the cost function decreases;

$-k \longleftarrow k+1$.

This algorithm can be used in shape optimization as well as for identification in inverse problems. The initialization of the domain gives the possibility to impose the zone where one wants to create holes or insert some obstacles.

Here we chose as initial guess the whole square $\Omega_{0}=\Omega$. Figure 6 shows the values of the topological gradient $\delta j$ computed on $\Omega$ :

$$
\delta j(x)=\left(u^{0}(x)-u_{i n j}\right) \cdot v^{0}(x), \quad \forall x \in \Omega .
$$

In the present example, we encounter the most favourable situation: the lowest value of the topological gradient coincides with the exact position of the optimal hole centre $X_{0}=(0.3,0.4)$, and consequently the solution to problem $(\mathcal{P})$ is obtained at the first iteration of Algorithm 1.

\subsubsection{Conclusion}

- Initial guess: a first difference between the two approaches described in Sections 4.1.1 and 4.1.2 is the initial guess. Unlike the classical shape optimization method (Sect. 4.1.1), the topological optimization method (Sect. 4.1.2) needs no initial guess. This property brings an interesting advantage to the second approach: it avoids the difficulties caused by the initial guess choice and its influence on the obtained results. Table 1 illustrates the dependence of the final result on the initial guess choice (see also Fig. 4).

- Iterations number: the second difference concerns the number of iterations. Table 1 gives the iterations and simulations numbers required by the first approach to obtain a good approximation of the solution. In the second approach, only one iteration was required. Of course, this is a particular example and we could not prove a general result concerning convergence rate comparison. However, fast convergence has been often observed 


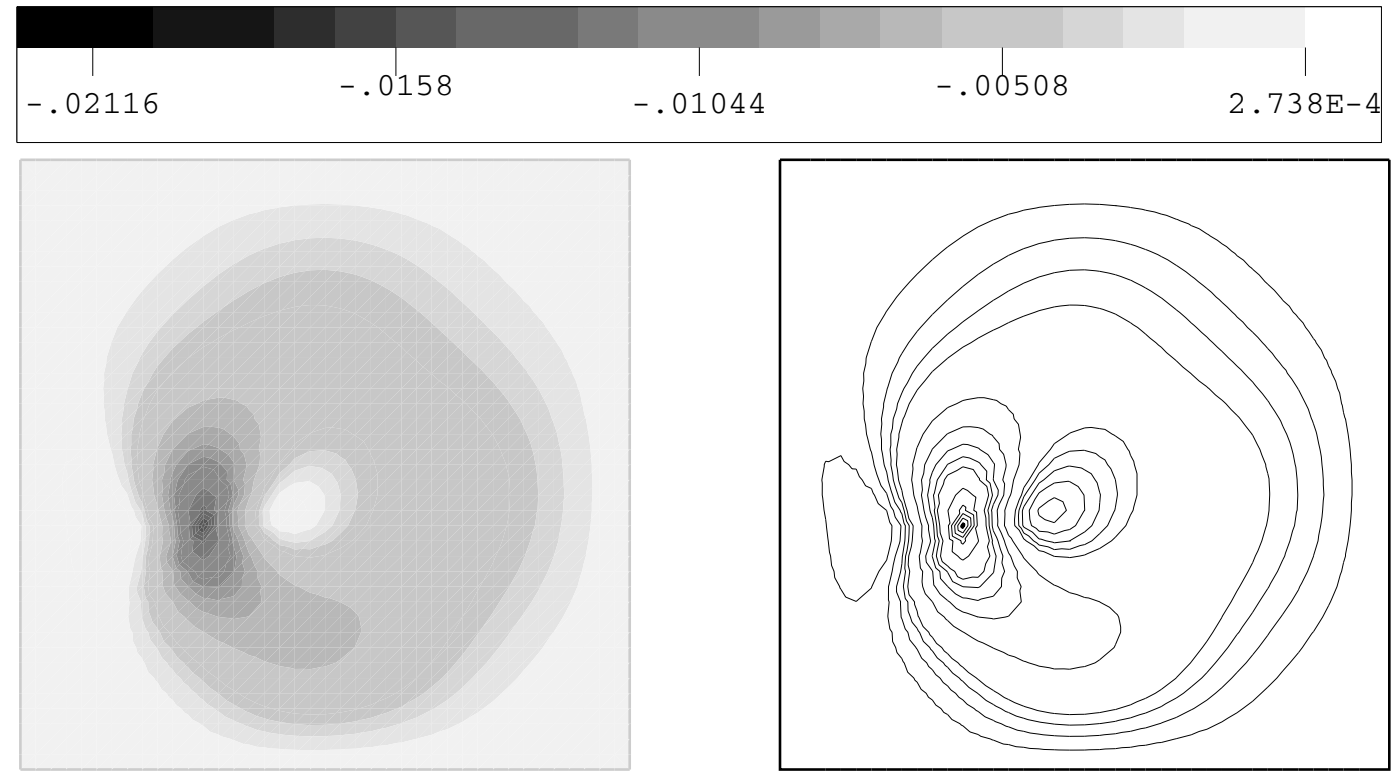

FiguRE 6. The topological gradient $g$.

in topological optimization and more particularly in topological identification of a finite number of holes; for other such examples, we refer the reader to $[13,17,23,25]$.

- Computational domain: this is a technical point which concerns implementation difficulties. In the first approach, the domain derivative and the associated systems (direct and adjoint problems) are computed in the perturbed domain $\Omega_{\varepsilon}$. In the second approach all computations are done in a fixed domain. It is well known that working in a variable domain needs introducing elaborated tools in order to take into account the domain shape and its deformations.

- Convergence: like all numerical approach, the topological gradient method has its own drawbacks. We have no convergence result for the numerical algorithm. Theoretically, the natural optimality condition is

$$
\delta j(x) \geq 0, \quad \text { for all } x \in \Omega \text {. }
$$

It coincides with the one obtained by Buttazzo and Dal Maso [10] for the Laplace equation using homogenization theory. But this condition has limited numerical application. In practice, some stop criterion can be successfully implemented, such as the material volume to be removed [13,17,22], the holes number to be inserted [25] or the obstacles number to be detected [23].

\subsection{Example 2: an improved version of topological gradient algorithm}

In this section we propose an improved version of the topological gradient Algorithm 1 presented in Section 4.1.2 (see also $[5,13,17,22,23,25]$ ). The new Algorithm 2 is based on the result given in Sections 3.1 and 3.3. It gives the possibility to create or to suppress holes during the optimization process. The numerical application that we consider is the water eutrophication problem [1], where several holes need to be inserted. In order to emphasize the efficiency of the new algorithm, we have compared the results obtained by using Algorithm 2 to those obtained by using Algorithm 1. In the first part of this section we describe the eutrophication problem and we introduce the mathematical model. Next, we present the three steps of the new algorithm. Numerical results are presented at the end of this section. 


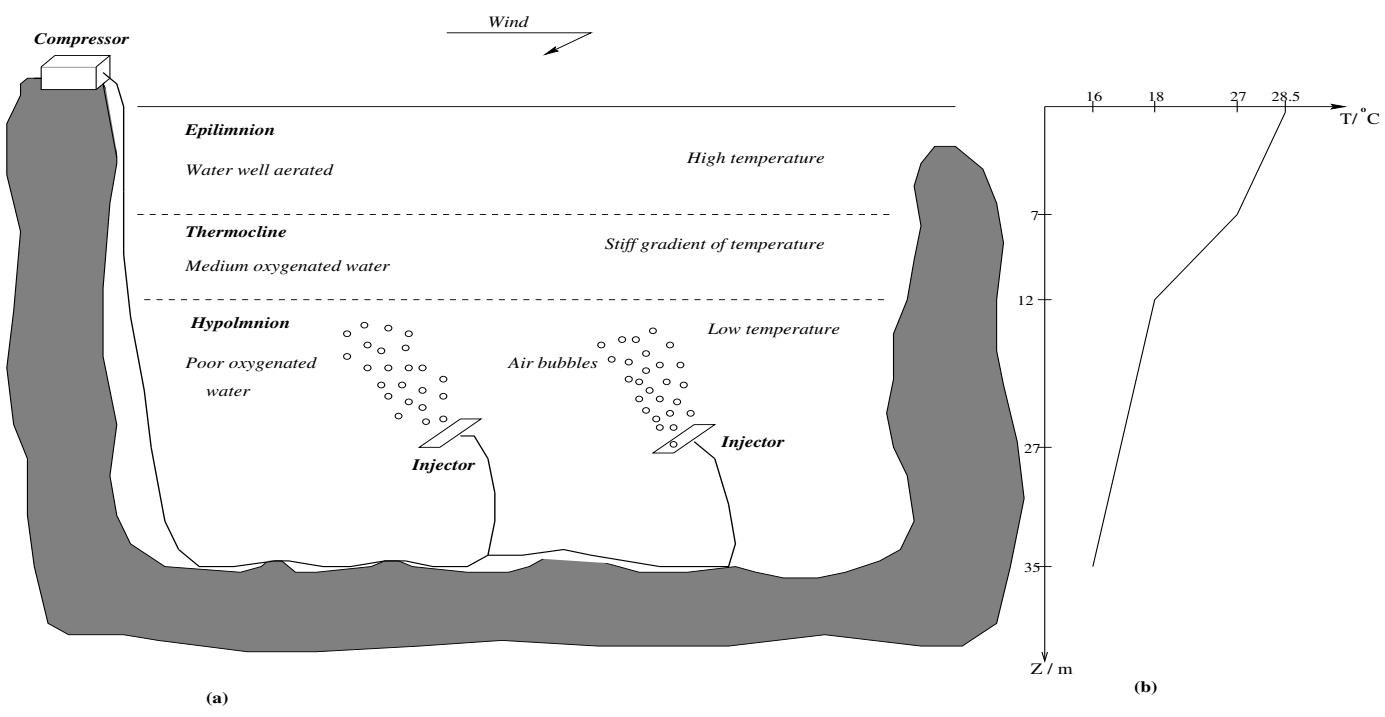

FiguRE 7. (a): Structure of a stratified lake; (b): average temperature curve during summer.

Eutrophication is a complex phenomena involving many physico-chemical parameters. Specifically in some climatic areas, the thermic factors combined to the biological and to the biochemical ones are dominant in the behavior of the aquatic ecosystems. Consequently, they generate important bio-climatology variations creating in lakes an unsteady dynamic process that decreases progressively water quality. Practically, the eutrophication in a water basin is characterized mainly by a poor dissolved oxygen concentration in water. Furthermore, this phenomena is accompanied by a stratification process dividing the water volume, during a large period of the year, into three distinct layers as depicted in Figure 7.

Three zones constitute this stratification:

(i) at the top, the epilimnion, a layer of around $7 \mathrm{~m}$ depth, well mixed by the effect of drafting wind and consequently well aerated;

(ii) in the middle, the thermocline, a zone with a quick decrease of temperature $\left(27^{\circ} \mathrm{C}\right.$ to $\left.18{ }^{\circ} \mathrm{C}\right)$ and of $5 \mathrm{~m}$ depth. This area is weakly affected by the wind action and consequently a medium rate of oxygen concentration is observed;

(iii) at the bottom, the hypolimnion, a deeper layer beyond $12 \mathrm{~m}$, having a temperature varying from $18{ }^{\circ} \mathrm{C}$ to $14^{\circ} \mathrm{C}$. This region is characterized by a low rate of oxygen and a high concentration of toxic gas $\left(\mathrm{H}_{2} \mathrm{~S}\right.$, ammoniac, carbonic gas, etc.)

The dynamic aeration process seems to be the most promising remedial technique to treat the water eutrophication phenomena. This technique consists in inserting air by the means of injectors located at the bottom of the lake in order to generate a vertical motion mixing up the water of the bottom with that in the top, thus oxygenating the lower part by bringing it in contact with the surface air.

Theoretically, the bubble flow is a multi-phase flow where the presence of free interfaces raises difficulties in the physical modelling as well as in the mathematical one. Hence, to obtain a physical and significant resolution by numerical simulation of the air injection phenomena in an eutrophised lake, one should consider a two-phase model: water-air bubble. This kind of modelling involves large systems of PDE's and variables in a multi-scale frame as well as closure conditions through turbulence model and phases interface interaction. Moreover, the 


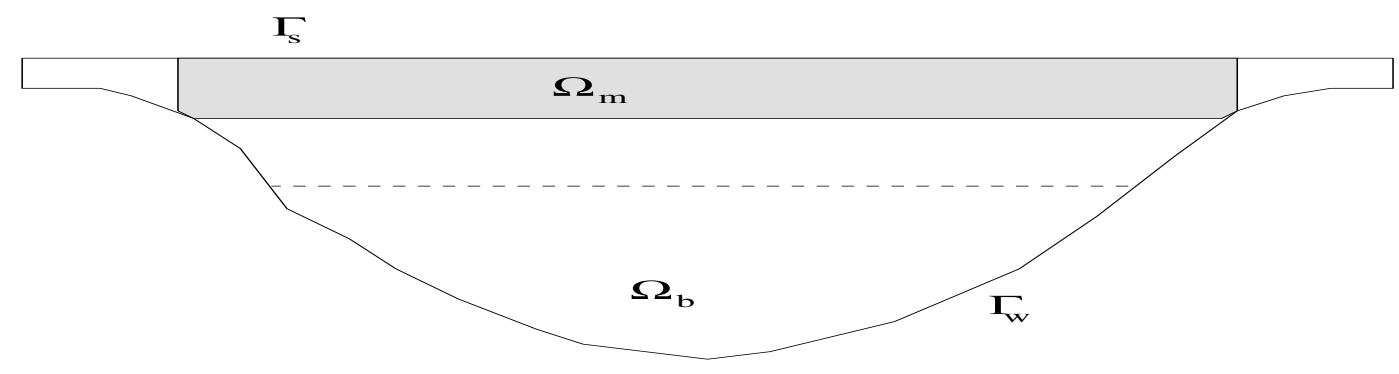

Figure 8. The geometry of the lake.

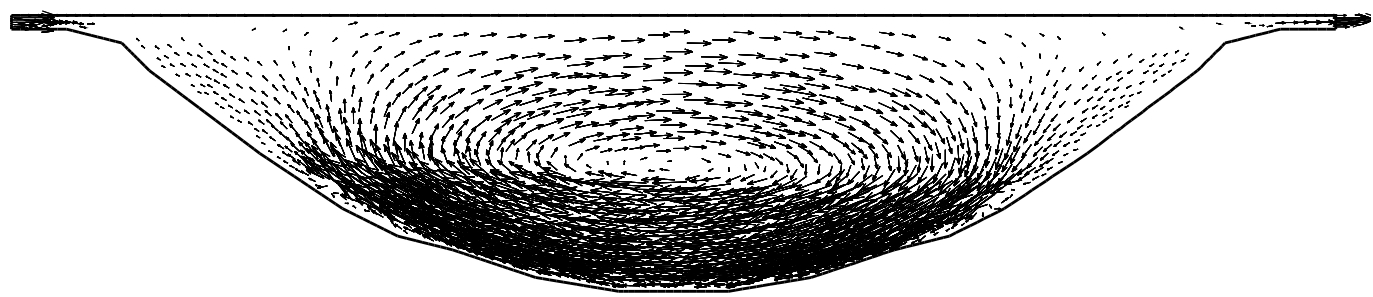

Figure 9. The wanted flow $\mathcal{U}_{g}$.

domain mesh size should be "small" in order to capture the significant variations of the spectrum. Therefore, the issue of the computational cost should be also addressed.

For all these reasons, we consider here, as a first approximation, only the liquid phase, which is the dominant one. The flow is described by a simplified model based on incompressible Stokes equations. The injected air is taken into account through local boundary conditions for the velocity on the injectors holes. We aim to optimize the injectors location in order to generate the best motion in the fluid with respect to the aeration purpose. The main idea is to compute the asymptotic topological expansion with respect to the insertion of an injector. In order to apply the theoretical previous results, each injector is modeled as a small hole $\omega_{\varepsilon}$ around a point $x_{0}$, having an injection velocity $\mathcal{U}_{i n j}$. The best locations and orientations are the ones for which the cost function decrease most, i.e. the sensitivity is as negative as possible.

Let $\Omega$ be a two dimensional flow domain representing the eutrophized water basin. The boundary $\Gamma$ of $\Omega$ consists in two parts (see Fig. 8): $\Gamma=\Gamma_{w} \cup \Gamma_{s}$ where

- $\Gamma_{w}$ is the bottom of the lake and/or eventually an open flow boundary (water entrance or exit),

- $\Gamma_{s}$ is the surface in contact with the atmosphere.

We suppose that a "good" lake oxygenation can be described by a target velocity $\mathcal{U}_{g}$. Then, the cost function $J$ which is here considered reads

$$
J(u)=\int_{\Omega_{m}}\left|u-\mathcal{U}_{g}\right|^{2} \mathrm{~d} x
$$

where $\Omega_{m} \subset \Omega$ is the measurement domain (the top layer, see Fig. 8). We aim to determine the optimal location in $\Omega_{b}$ (the bottom of the lake, see Fig. 8) of some injector holes $\omega_{k}$ in order to minimize function $J$. 


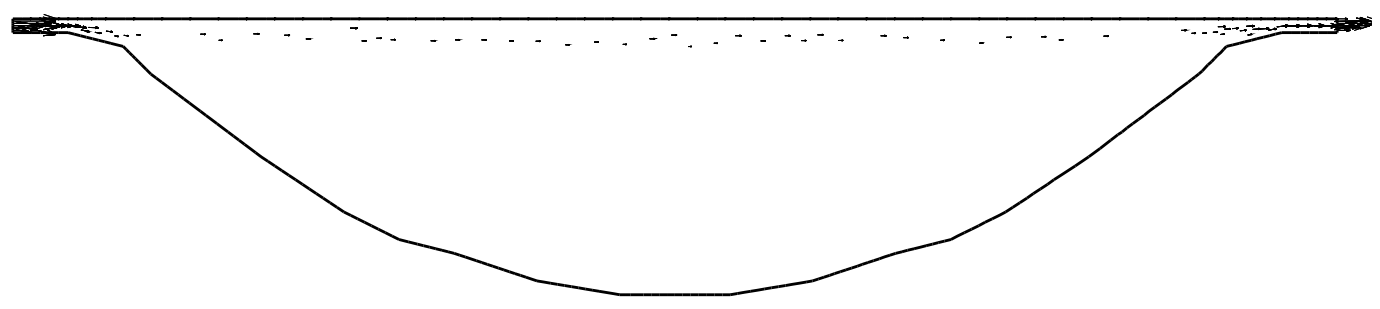

Figure 10. The initial flow $u^{0}$.

Recall that we consider Stokes equations with a non homogeneous boundary condition on $\partial \omega_{\varepsilon}\left(u_{D \mid \partial \omega_{\varepsilon}}^{\varepsilon}=\right.$ $\left.\mathcal{U}_{i n j}\right)$. In this case, the topological sensitivity $\delta j$ is given by

$$
\delta j(x)=\left(u^{0}(x)-\mathcal{U}_{i n j}\right) \cdot v^{0}(x) \quad \forall x \in \Omega
$$

where $u^{0}$ and $v^{0}$ are respectively solution to

$$
\begin{aligned}
& \left\{\begin{aligned}
\nu \Delta u^{0}+\nabla p^{0} & =0 & & \text { in } \Omega \\
\operatorname{div} u^{0} & =0 & & \text { in } \Omega \\
u^{0} & =u_{w} & & \text { in } \Gamma_{s} \\
u^{0} & =0 & & \text { in } \Gamma_{w}
\end{aligned}\right. \\
& \left\{\begin{aligned}
\nu \Delta v^{0}+\nabla q^{0} & =-2\left(u^{0}-\mathcal{U}_{g}\right) \chi_{\Omega_{m}} & & \text { in } \Omega \\
\operatorname{div} v^{0} & =0 & & \text { in } \Omega \\
v^{0} & =0 & & \text { in } \Gamma_{s} \\
v^{0} & =0 & & \text { in } \Gamma_{w} .
\end{aligned}\right.
\end{aligned}
$$

Here $u_{w}$ is a given velocity (wind velocity) and $\chi_{\Omega_{m}}$ is the characteristic function of the measurement domain.

Numerical implementation: We use a fixed triangular mesh. The topological gradient $\delta j$ is computed at each mesh node. The center $x_{0}$ of the hole $\omega_{\varepsilon}=x_{0}+\varepsilon \omega$ which will be created is deduced as the node where $\delta j$ is the most negative. The numerical algorithm is based on the result given in Sections 3.1 and 3.3. A new optimization algorithm is proposed, giving the possibility to create or to suppress holes during the optimization process. It proceeds by iterations. At the $k$-th iteration, $\delta j^{k}$ denotes the topological gradient and $\Omega_{k}$ denotes the current domain. We denote by $x_{p}^{k}, p=1,2, \ldots$, the local minimum of $\delta j^{k}$. The set of holes which are candidates to be inserted in $\Omega_{k}$ is given by

$$
h^{k}=\left\{\omega_{\varepsilon}(x)=x+\varepsilon \omega, x \in C_{k}\right\}
$$

where $C_{k}=\left\{x_{p}^{k} \in \Omega_{k}, 1 \leq p \leq n_{k}\right\}$ is the set of the negative local minimum of $\delta j^{k}$ in $\Omega_{k}$ and $n_{k}$ is their number (supposed to be finite, which is the case for the discrete problem). An alternative to this choice would be to consider instead a set of the form $C_{k}=\left\{x \in \Omega_{k}, \delta j^{k}(x) \leq L_{k}\right\}$ where the level $L_{k}$ is such that mes $\left(C_{k}\right)$ is a given fraction of the total volume. The holes are ordered in such a way that $\delta j^{k}\left(x_{p}^{k}\right) \leq \delta j^{k}\left(x_{p^{\prime}}^{k}\right)$ for all $1 \leq p<p^{\prime} \leq n_{k}$.

Let $H^{k}$ be the set of $N_{k}$ holes inserted during all previous iterations:

$$
H^{k}=\left\{\omega_{\varepsilon}\left(y_{l}^{k}\right)=y_{l}^{k}+\varepsilon \omega, 1 \leq l \leq N_{k}\right\}
$$


The variation of the cost function with respect to suppression of hole $y_{l}^{k}+\varepsilon \omega$ is denoted by $g_{l}^{k}=g^{k}\left(y_{l}^{k}\right)$ and the set $\left\{y_{l}^{k}, 1 \leq l \leq N_{k}\right\}$ is supposed to be arranged in such a way that $g_{l}^{k} \leq g_{l^{\prime}}^{k}$ if $l<l^{\prime}$.

The proposed algorithm is based on three steps. The first step solves the partial differential equations (56)(57), computes the topological gradient and derives the set of holes which are candidate to be inserted.

The second step introduces the possibility of suppressing existing holes and inserting new ones. Consequently, the cost function can be decreased without changing the number of holes. For this, the variation $\delta j_{i}^{k}$ of the cost function when creating a new hole $\omega_{\varepsilon}\left(x_{i}^{k}\right)$ is compared to the variation $g_{i}^{k}$ of the cost function when removing an existing hole $\omega_{\varepsilon}\left(y_{i}^{k}\right)$. In order to decrease the cost function, one suppresses $\omega_{\varepsilon}\left(y_{i}^{k}\right)$ and inserts $\omega_{\varepsilon}\left(x_{i}^{k}\right)$ only when the variation $\delta j_{i}^{k}+g_{i}^{k}$ is negative.

The last step inserts some new holes. The number of holes added at the $k$-th iteration is denoted $m_{k}$.

\section{Algorithm 2.}

- Initialization: choose $\Omega_{0}=\Omega_{b}$, and set $k=0$.

- Repeat until $\delta j^{k} \geq 0$ in $\Omega_{k}$ :

- Step 1: preparation phase

* solve (56) and (57) in $\Omega_{k}$;

* compute the topological sensitivity $\delta j^{k}$;

* determine the set $h^{k}=\left\{\omega_{\varepsilon}\left(x_{p}^{k}\right)=x_{p}^{k}+\varepsilon \omega, 1 \leq p \leq n_{k}\right\}$.

- Step 2: exchange phase

* compute the variations $\left\{g_{l}^{k}=g^{k}\left(y_{l}^{k}\right), 1 \leq l \leq N_{k}\right\}$;

* set $q=1$;

* while the variation $\delta j^{k}\left(x_{q}^{k}\right)+g^{k}\left(y_{q}^{k}\right)<0$ :

- remove the hole $\omega_{\varepsilon}\left(y_{q}^{k}\right)=y_{q}^{k}+\varepsilon \omega$ and add the hole $\omega_{\varepsilon}\left(x_{q}^{k}\right)=x_{q}^{k}+\varepsilon \omega$;

- increment $q$,

- Step 3: insertion phase

* creation of the holes $\left\{x_{q+i}^{k}+\varepsilon \omega, 1 \leq i \leq m_{k}\right\}$, where $q$ is the number of the holes changed during the second step;

* set $\Omega_{k+1}=\Omega_{k} \cup\left(\cup_{i=1}^{q} \omega_{\varepsilon}\left(y_{i}^{k}\right)\right) \backslash\left(\cup_{l=1}^{q+m_{k}} \overline{\omega_{\varepsilon}\left(x_{l}^{k}\right)}\right)$ where $\left\{\omega_{\varepsilon}\left(y_{i}^{k}\right), 1 \leq i \leq q\right\}$ are the holes removed during the second step,

- increment $k$.

In the above algorithm, the systems (56) and (57) are discretized by a finite element method $[9,15]$. The computation of the approximate solution is achieved by Uzawa algorithm.

In the context of water eutrophication, each hole $\omega_{\varepsilon}\left(x_{i}\right)=x_{i}+\varepsilon \omega$ is replaced by an injector located at the point $x_{i}$. A constant number of injectors were added at each iteration (one or five at each iteration).

In order to test the advantage of this approach, we have compared the results issued from this technique to those obtained by using a classical version as described in $[17,22,23,25]$, that is, without the second step. The obtained results are presented in Figures 11-16. Figure 11 describes the variation of the cost function during the optimization process when adding one injector at each iteration. We compare the values of $J$ obtained using Algorithm 1 (dashed line) to those obtained using Algorithm 2 (continuous line). One can remark that the value of $J$ is smaller when using Algorithm 2, which can be interpreted by the good distribution of the obtained injectors locations. Figure 12 illustrates the variation of the same function when adding five injectors at each iteration. The injectors locations obtained during the optimization process are presented in Figure 13 when adding one injector at each iteration and in Figure 14 when adding five injectors at each iteration. Both figures 


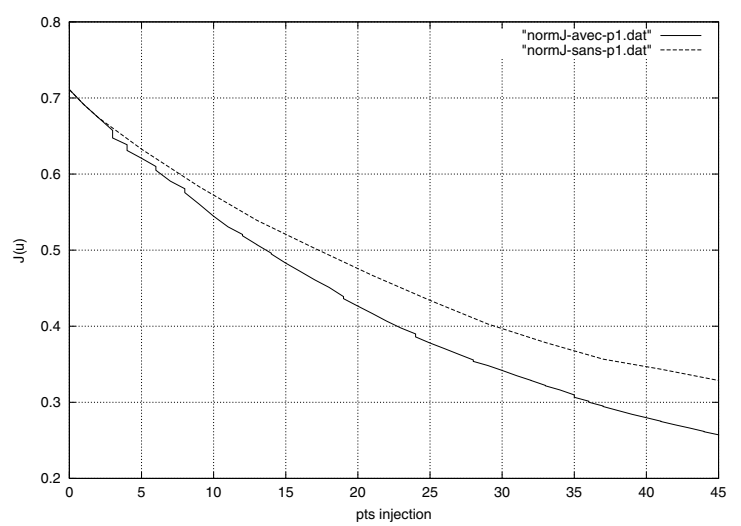

FiguRE 11. Variation of the cost function when adding one injector at each iteration; without removing injectors (top), with removing (bottom).

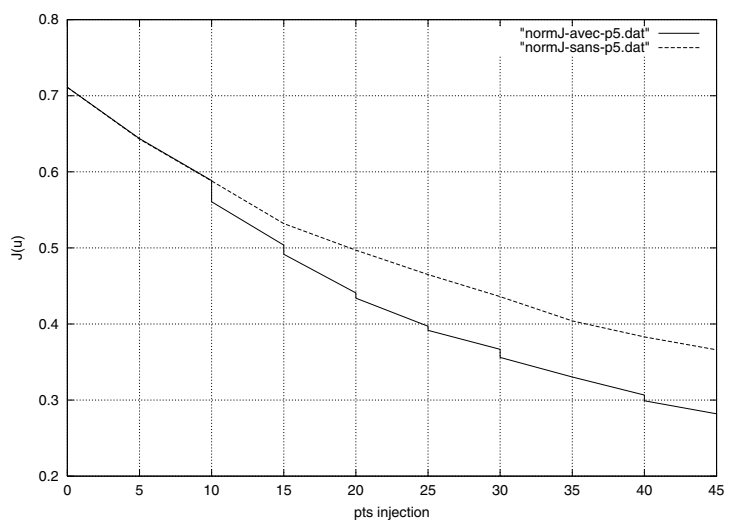

FIGURE 12. Variation of the cost function when adding five injectors at each iteration; without removing injectors (top), with removing (bottom).

show the locations obtained when removing injectors (right) and those obtained without removing injectors (left). Figure 15 presents the wanted velocity $\mathcal{U}_{g}$ and the obtained velocities in the measurement $\Omega_{m}$ when the optimization process is achieved. The given velocities are denoted as follow:

- $u_{1}$ is the velocity obtained by using a classical algorithm (without removing phase) and adding one injector at each iteration;

- $u_{2}$ is the velocity obtained by using the new approach and adding one injector at each iteration;

- $u_{3}$ is the velocity obtained by using a classical algorithm (without removing phase) and adding five injectors at each iteration;

- $u_{4}$ is the velocity obtained by using the new approach and adding five injector at each iteration.

To facilitate the comparison with the wanted velocity $\mathcal{U}_{g}$, we illustrate in Figure 16 the isolines of $\left\|u_{i}-\mathcal{U}_{g}\right\|$, $1 \leq i \leq 4$. Isolines $1,2,3$, and 4 correspond respectively to isovalues $0,0.001,0.01$ and 0.1 . In the two considered cases (when adding one injector (Figs. 16a and 16b) or five injectors (Figs. 16c and 16d), one can observe that the obtained velocities using Algorithm 2 are more close to the wanted velocity $\mathcal{U}_{g}$ than those obtained using Algorithm 1. Such a comparison between the results computed with (Algorithm 2) and without (Algorithm 1) removing injectors show clearly the important effect of the exchange step (second step). 


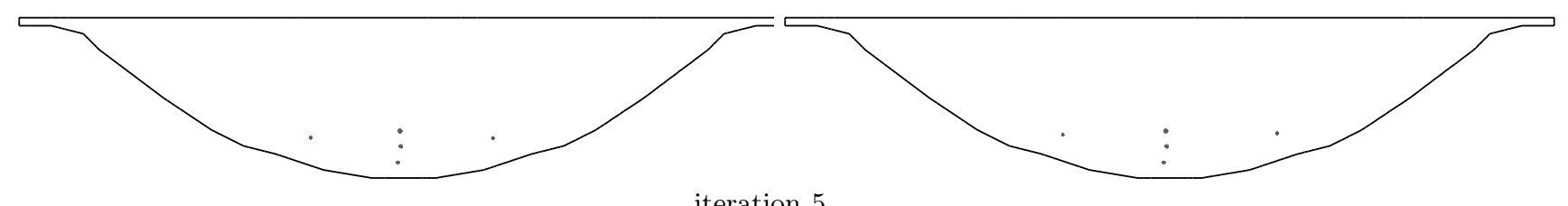

iteration 5 .
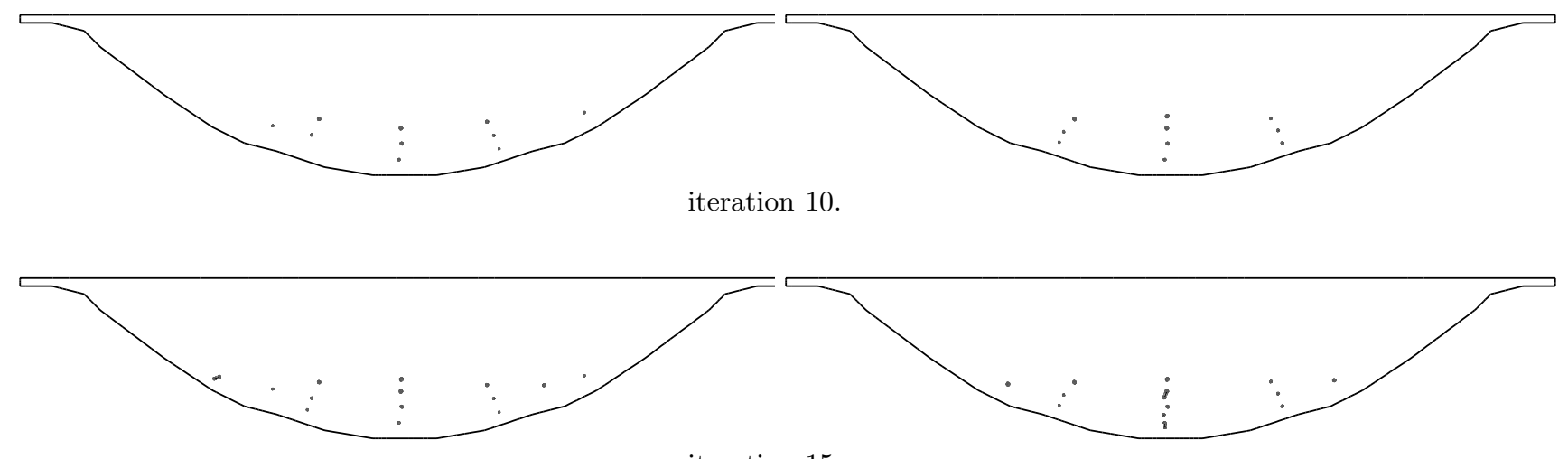

iteration 15 .

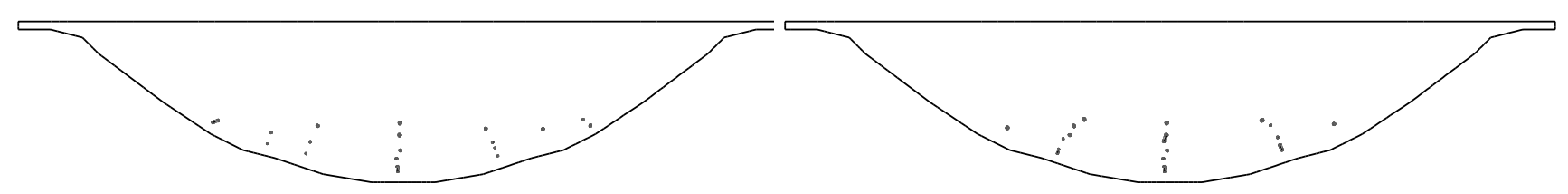

iteration 20 .

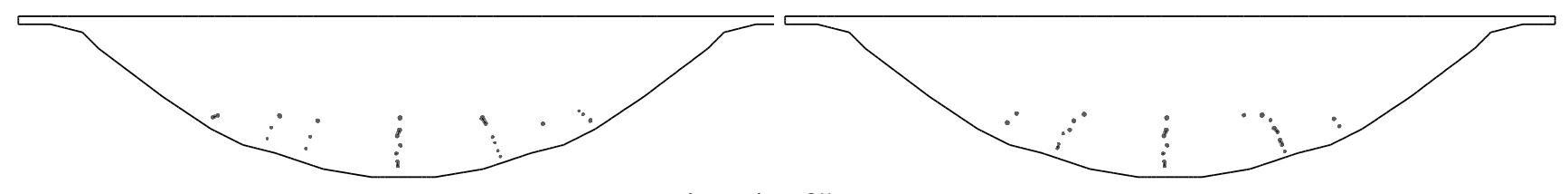

iteration 25 .

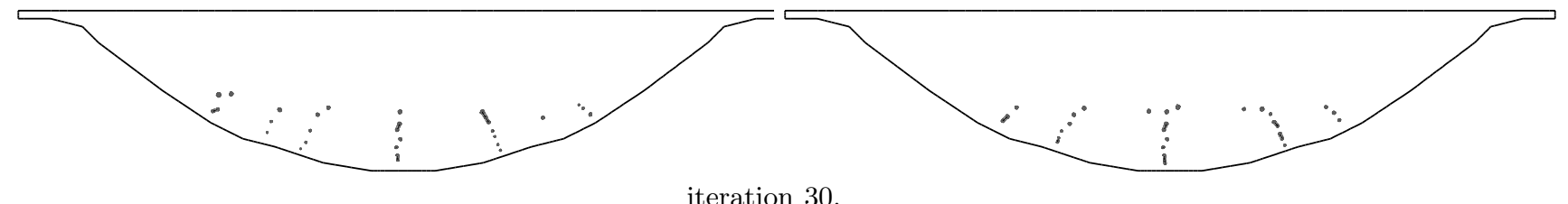

(a) Without removing injectors (Algorithm 1).

(b) With removing injectors (Algorithm 2).

FIGURE 13. Injectors locations obtained during the optimization process by adding one injector at each iteration. 


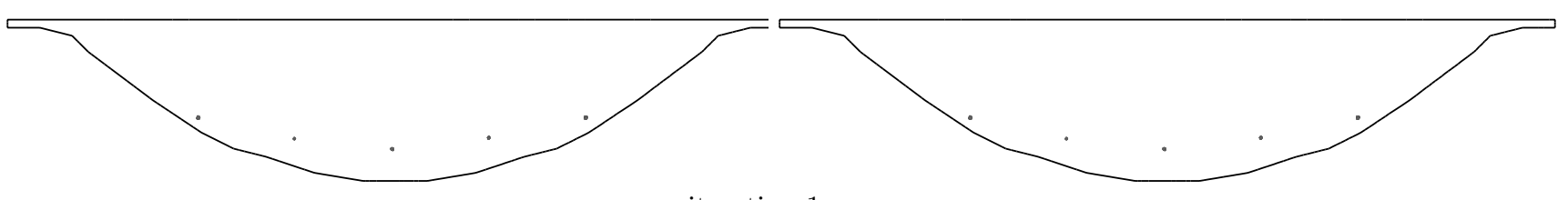

iteration 1.

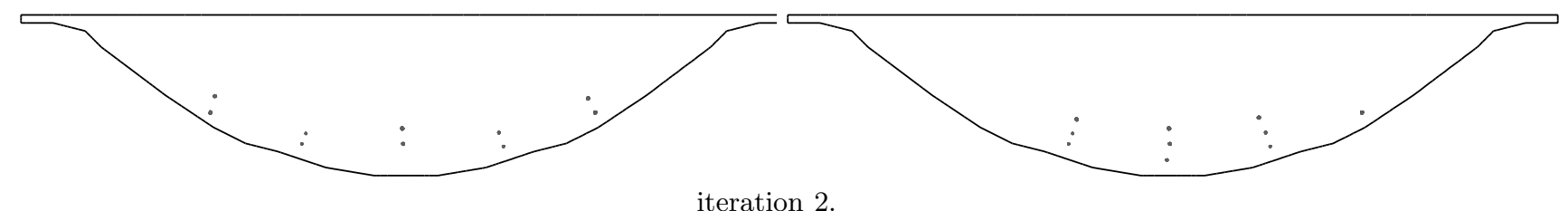

iteration 2 .

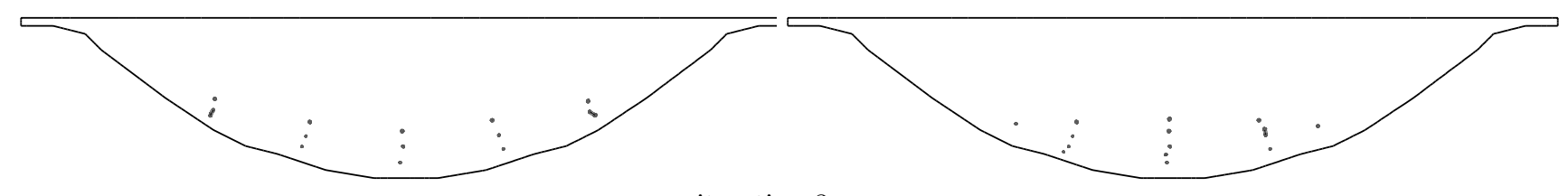

iteration 3.

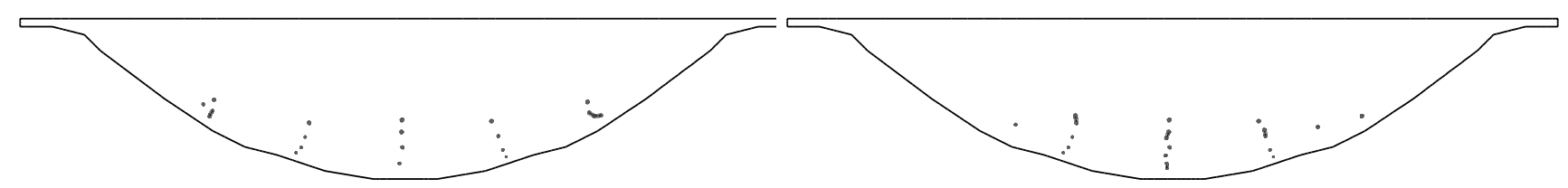

iteration 4.

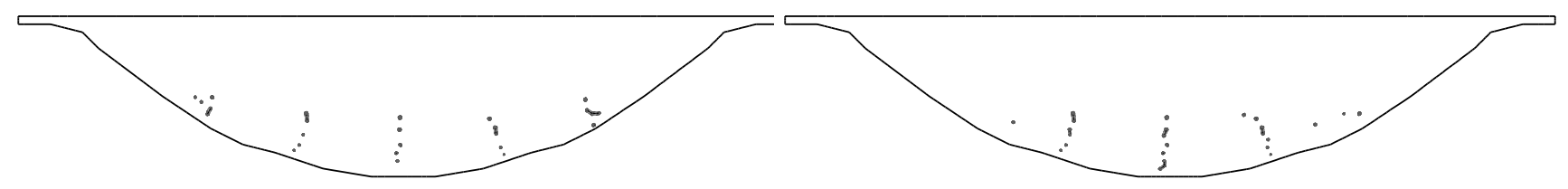

iteration 5.

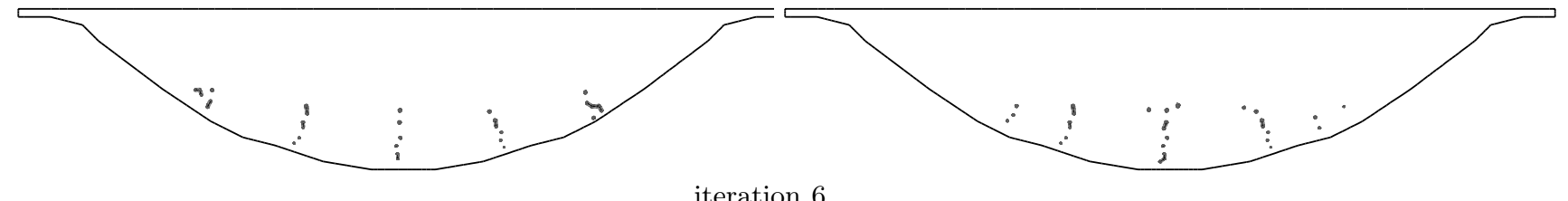

(a) Without removing injectors (Algorithm 1).

(b) With removing injectors (Algorithm 2).

FIGURE 14. Injectors locations obtained during the optimization process by adding five injectors at each iteration. 


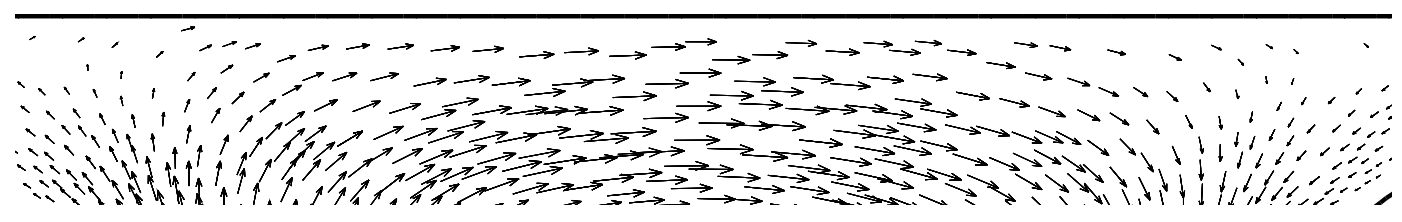

The wanted velocity $\mathcal{U}_{g}$ in the measurement domain $\Omega_{m}$.

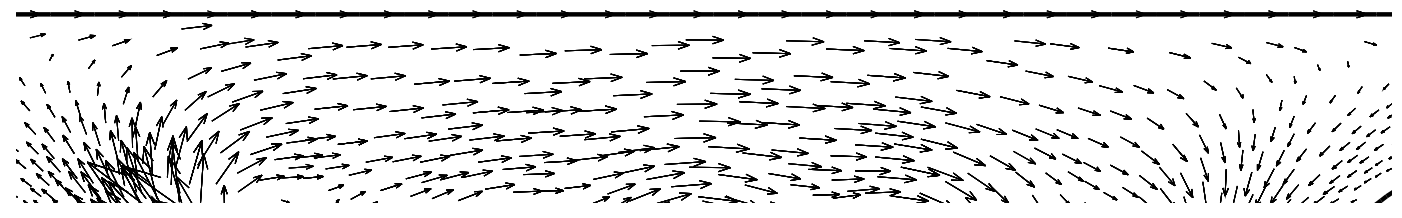

(a) The obtained velocity $u_{1 \mid \Omega_{m}}$ (without removing, adding one injector at each iteration).

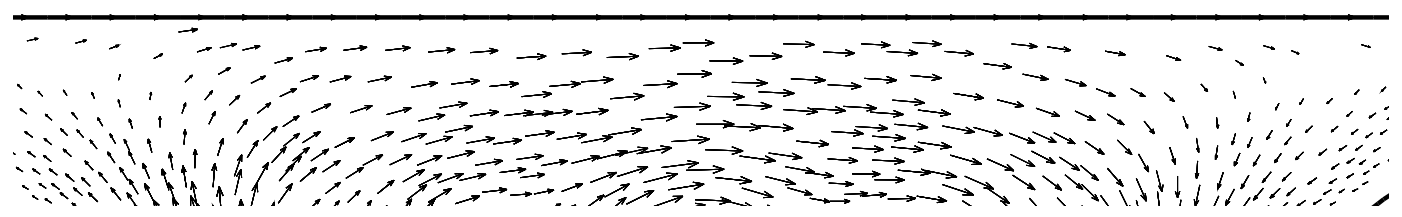

(b) The obtained velocity $u_{2 \mid \Omega_{m}}$ (with removing, adding one injector at each iteration).

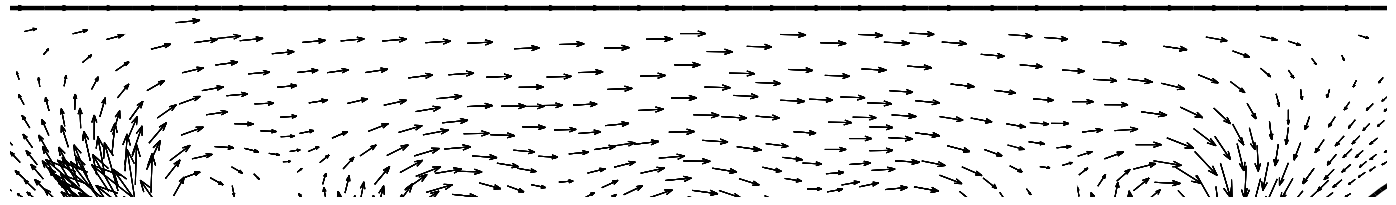

(c) The obtained velocity $u_{3 \mid \Omega_{m}}$ (without removing, adding five injectors at each iteration).

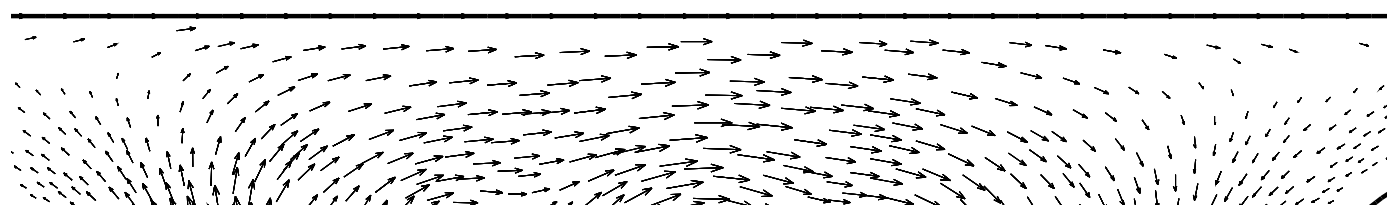

(d) The obtained velocity $u_{1 \mid \Omega_{m}}$ (with removing, adding five injectors at each iteration).

FIGURE 15. Velocities fields obtained in $\Omega_{m}$ (measurement domain) at the end of the optimization process. 


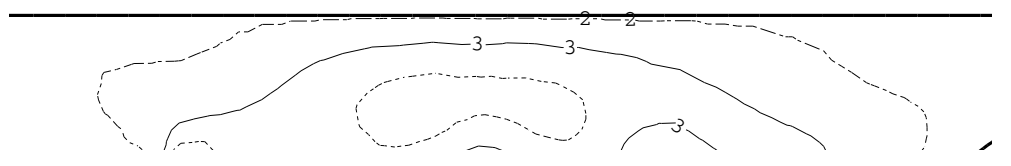

(a) Iso-lignes of $\left\|u_{1}-\mathcal{U}_{g}\right\|$ (without removing, adding one injector at each iteration).

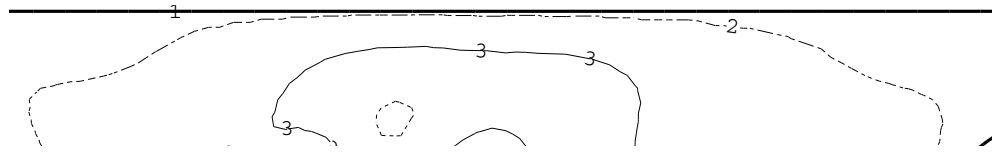

(b) Iso-lignes of $\left\|u_{2}-\mathcal{U}_{g}\right\|$ (with removing, adding one injector at each iteration).

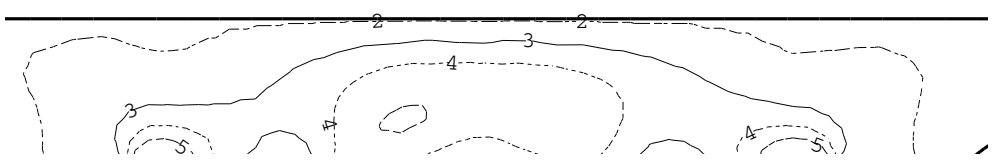

(c) Iso-lignes of $\left\|u_{3}-\mathcal{U}_{g}\right\|$ (without removing, adding five injectors at each iteration).

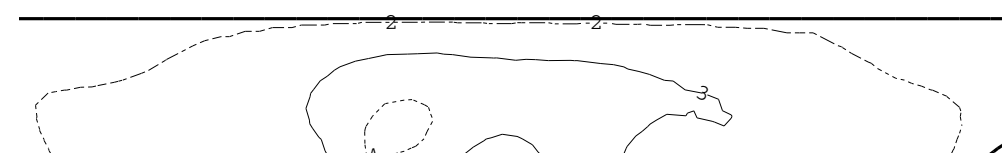

(d) Iso-lignes of $\left\|u_{4}-\mathcal{U}_{g}\right\|$ (with removing, adding five injectors at each iteration).

FIGURE 16. Iso-lignes of $\left\|u_{i}-\mathcal{U}_{g}\right\|, 1 \leq i \leq 4$ ( $1=$ isoline $0.0,2=$ isoline $0.001,3=$ isoline 0.01 and $4=$ isoline 0.02 ). 


\section{REFERENCES}

[1] M. Abdelwahed, M. Amara, F. El Dabaghi and M. Hassine, A numerical modelling of a two phase flow for water eutrophication problems. ECCOMAS 2000, European Congress on Computational Methods in Applied Sciences and Engineering, Barcelone, 11-14 September (2000).

[2] G. Allaire and A. Henrot, On some recent advances in shape optimization. C. R. Acad. Sci. Paris, Ser. II B 329 (2001) 383-396.

[3] G. Allaire and R. Kohn, Optimal bounds on the effective behavior of a mixture of two well-orded elastic materials. Quart. Appl. Math. 51 (1996) 643-674.

[4] G. Allaire, F. Jouve and A.-M. Toader, Structural optimization using sensitivity analysis and a level-set method. J. Comput. Phys. 194 (2004) 363-393.

[5] S. Amstutz, M. Masmoudi and B. Samet, The topological asymptotic for the Helmholtz equation. SIAM J. Contr. Optim. 42 (2003) 1523-1544.

[6] J.A. Bello, E. Fernández-Cara, J. Lemoine and J. Simon, The differentiability of the drag with respect to the variations of a lipschitz domain in a Navier-Stokes flow. SIAM J. Control Optim. 35 (1997) 626-640.

[7] M. Bendsoe, Optimal topology design of continuum structure: an introduction. Technical report, Departement of mathematics, Technical University of Denmark, DK2800 Lyngby, Denmark (1996).

[8] M. Bendsoe, N. Olhoff and O. Sigmund, IUTAM Symposium on Topological Design Optimization of Structures, Machines and Materials. Springer (2006).

[9] F. Brezzi and M. Fortin, Mixed and hybrid finite element method, Springer Series in Computational Mathematics 15. Springer Verlag- New York (1991)

[10] G. Buttazzo and G. Dal Maso, Shape optimization for Dirichlet problems: Relaxed formulation and optimality conditions. Appl. Math. Optim. 23 (1991) 17-49.

[11] J. Céa, Conception optimale ou identification de forme, calcul rapide de la dérivée directionnelle de la fonction coút. RAIRO Math. Modél. Anal. Numér. 20 (1986) 371-402.

[12] J. Céa, A. Gioan and J. Michel, Quelques résultats sur l'identification de domains. Calcolo (1973).

[13] J. Céa, S. Garreau, P. Guillaume and M. Masmoudi, The shape and topological optimizations connection. Comput. Methods Appl. Mech. Engrg. 188 (2000) 713-726.

[14] M. Chipot and G. Dal Maso, Relaxed shape optimization: the case of nonnegative data for the Dirichlet problems. Adv. Math. Sci. Appl. 1 (1992) 47-81.

[15] P. Ciarlet, The Finite Element Method for Elliptic Problems. North-Holland (1978).

[16] R. Dautray and J. Lions, Analyse mathémathique et calcul numérique pour les sciences et les techniques. Masson, collection CEA (1987).

[17] S. Garreau, P. Guillaume and M. Masmoudi, The topological asymptotic for pde systems: the elasticity case. SIAM J. Control Optim. 39 (2001) 1756-1778.

[18] V. Girault and P.A. Raviart, Finite element methods for Navier-Stokes equations, Theory and Algorithms. Springer Verlag (1986).

[19] R. Glowinski and O. Pironneau, Toward the computational of minimun drag profile in viscous laminar flow. Appl. Math. Model. 1 (1976) 58-66.

[20] P. Guillaume, Dérivées d'ordre supérieur en conception optimale de forme. Ph.D. thesis, Université Paul Sabatier, Toulouse, France (1994).

[21] P. Guillaume and M. Masmoudi, Computation of high order derivatives in optimal shape design. Numer. Math. 67 (1994) 231-250.

[22] P. Guillaume and K. Sid Idris, The topological asymptotic expansion for the Dirichlet Problem. SIAM J. Control. Optim. 41 (2002) 1052-1072.

[23] P. Guillaume and K. Sid Idris, Topological sensitivity and shape optimization for the Stokes equations. SIAM J. Control Optim. 43 (2004) 1-31.

[24] M.D. Gunzburger and H. Kim, Existence of an optimal solution of a shape control problem for the stationary Navier-Stokes equations. SIAM J. Control Optim. 36 (1998) 895-909.

[25] M. Hassine and M. Masmoudi, The topological sensitivity analysis for the Quasi-Stokes problem. ESAIM: COCV 10 (2004) $478-504$.

[26] J. Jacobsen, N. Olhoff and E. Ronholt, Generalized shape optimization of three-dimensionnal structures using materials with optimum microstructures. Technical report, Institute of Mechanical Engineering, Aalborg University, DK-9920 Aalborg, Denmark (1996).

[27] J.L. Lions and E. Magenes, Problèmes aux limites non homogenes et applications. Dunod (1968).

[28] M. Masmoudi, The topological asymptotic, in Computational Methods for Control Applications, H. Kawarada and J. Periaux Eds., International Séries Gakuto (2002).

[29] M. Masmoudi, J. Pommier and B. Samet, The topological asymptotic expansion for the Maxwell equations and some applications. Inverse Probl. 21 (2005) 547-564. 
[30] V. Mazja, S.A. Nazarov and B.A. Plamenevski, Asymptotic theory of elliptic boundary value problems in singularly perturbed domains. Vol. I, Birkhäuser (2000).

[31] O. Pironneau, On optimum profiles in Stokes flow. J. Fluid Mech. 59 (1973) 117-128.

[32] O. Pironneau, Optimal Shape Design for Elliptic Systems. Springer, Berlin (1984).

[33] A. Schumacher, Topologieoptimierung von bauteilstrukturen unter verwendung von lopchpositionierungkrieterien. $\mathrm{Ph} . \mathrm{D}$. thesis, Universitat-Gesamthochschule-Siegen (1995).

[34] M. Shœnauer, L. Kallel and F. Jouve, Mechanical inclusions identification by evolutionary computation. Revue européenne des éléments finis $\mathbf{5}$ (1996) 619-648.

[35] J. Simon, Domain variation for Stokes flow, in Lect. Notes Control Inform. Sci. 159, X. Li and J. Yang Eds., Springer, Berlin (1990) 28-42.

[36] J. Simon, Domain variation for drag Stokes flows, in Lect. Notes Control Inform. Sci. 114, A. Bermudez Eds., Springer, Berlin (1987) $277-283$.

[37] J. Sokolowski and A. Zochowski, On the topological derivative in shape optimization. SIAM J. Control Optim. 37 (1999) 1251-1272 (electronic)

[38] J. Sokolowski and A. Zochowski, Modelling of topological derivatives for contact problems. Numer. Math. 102 (2005) $145-179$.

[39] R. Temam, Navier Stokes equations (1985). 